\section{OPEN ACCESS}

Edited by:

Mikhail Lebedev,

Duke University, United States

Reviewed by:

Gang Zhang,

Institute of High Performance Computing ( $A^{*}$ STAR), Singapore Carlos Vicario-Abejón, Consejo Superior de Investigaciones

Cientificas (CSIC), Spain

*Correspondence:

Mattia Bramini

mattia.bramini@iit.it

Fabrizia Cesca

fabrizia.cesca@iit.it

tSenior authors.

Received: 31 January 2018 Accepted: 26 March 2018

Published: 11 April 2018

Citation:

Bramini M, Alberini G, Colombo E, Chiacchiaretta M, DiFrancesco ML, Maya-Vetencourt JF, Maragliano L, Benfenati $F$ and Cesca $F$ (2018) Interfacing Graphene-Based Materials With Neural Cells.

Front. Syst. Neurosci. 12:12. doi: 10.3389/fnsys.2018.00012

\title{
Interfacing Graphene-Based Materials With Neural Cells
}

\begin{abstract}
Mattia Bramini ${ }^{1,2 *}$, Giulio Alberini ${ }^{1,3}$, Elisabetta Colombo ${ }^{1,2}$, Martina Chiacchiaretta $^{1,3}$, Mattia L. DiFrancesco ${ }^{1,2}$, José F. Maya-Vetencourt ${ }^{1}$, Luca Maragliano ${ }^{1}$, Fabio Benfenati ${ }^{1,2,3+}$ and Fabrizia Cesca ${ }^{1,2 *}$
\end{abstract}

${ }^{1}$ Center for Synaptic Neuroscience and Technology, Istituto Italiano di Tecnologia, Genova, Italy, ${ }^{2}$ Graphene Labs, Istituto Italiano di Tecnologia, Genova, Italy, ${ }^{3}$ Department of Experimental Medicine, Università degli Studi di Genova, Genova, Italy

The scientific community has witnessed an exponential increase in the applications of graphene and graphene-based materials in a wide range of fields, from engineering to electronics to biotechnologies and biomedical applications. For what concerns neuroscience, the interest raised by these materials is two-fold. On one side, nanosheets made of graphene or graphene derivatives (graphene oxide, or its reduced form) can be used as carriers for drug delivery. Here, an important aspect is to evaluate their toxicity, which strongly depends on flake composition, chemical functionalization and dimensions. On the other side, graphene can be exploited as a substrate for tissue engineering. In this case, conductivity is probably the most relevant amongst the various properties of the different graphene materials, as it may allow to instruct and interrogate neural networks, as well as to drive neural growth and differentiation, which holds a great potential in regenerative medicine. In this review, we try to give a comprehensive view of the accomplishments and new challenges of the field, as well as which in our view are the most exciting directions to take in the immediate future. These include the need to engineer multifunctional nanoparticles (NPs) able to cross the blood-brain-barrier to reach neural cells, and to achieve on-demand delivery of specific drugs. We describe the state-of-the-art in the use of graphene materials to engineer three-dimensional scaffolds to drive neuronal growth and regeneration in vivo, and the possibility of using graphene as a component of hybrid composites/multi-layer organic electronics devices. Last but not least, we address the need of an accurate theoretical modeling of the interface between graphene and biological material, by modeling the interaction of graphene with proteins and cell membranes at the nanoscale, and describing the physical mechanism(s) of charge transfer by which the various graphene materials can influence the excitability and physiology of neural cells.

Keywords: graphene, neurology, brain, blood-brain barrier, nanomedicine, scaffolds, smart materials, computational modeling

\section{INTRODUCTION}

Graphene $(\mathrm{G})$ is a single- or few-layered sheet of $\mathrm{Sp}^{2}$-bonded carbon atoms tightly packed in a two-dimensional (2D) honeycomb lattice, with a thickness of only $0.34 \mathrm{~nm}$ (Geim, 2009). Each carbon atom has three $\mu$-bonds and an out-of-plane $\pi$-bond that can bind with neighboring atoms (Geim, 2009), making G the thinnest compound ever known at one atom thick and the 
strongest compound discovered. Moreover, it is light, flexible and transparent and both electrically and thermally highly conductive, which opens the possibility of using it in a broad spectrum of applications, including supercapacitors (Hess et al., 2011; Sahoo et al., 2015; Casaluci et al., 2016), flexible electronics (Eda et al., 2008; Meric et al., 2008), printable inks (Zhu et al., 2015; Bonaccorso et al., 2016), batteries (Hassoun et al., 2014; Dufficy et al., 2015), optical and electrochemical sensors (Pumera, 2009; Du et al., 2010; Kang et al., 2010), energy storage (El-Kady and Kaner, 2013; Bonaccorso et al., 2015; Ambrosi and Pumera, 2016) and medicine (Novoselov et al., 2012; Casaluci et al., 2016; Kostarelos et al., 2017; Reina et al., 2017). G-related materials (GRMs) include single- and few-layered G (1-10 layers; GR), G oxide (single layer, 1:1 C/O ratio; GO), reduced G oxide (rGO), graphite nano- and micro-platelets (more than 10 layers, but $<100 \mathrm{~nm}$ thickness and average lateral size in the order of the $\mathrm{nm}$ and $\mu \mathrm{m}$, respectively), $\mathrm{G}$ and $\mathrm{G}$ oxide quantum dots (GQDs and GOQDs, respectively), and a variety of hybridized G nanocomposites (Bianco, 2013; Wick et al., 2014; Cheng et al., 2016). Having such different composition and structures, these compounds possess very diverse properties that have to be taken in consideration when planning biomedical applications, as they elicit completely different biological responses. Thus, it is fundamental to properly identify and characterize the GRMs employed, to overcome the widespread lack of reproducibility affecting biological experiments with $G$ materials.

In the last few years, biomedical applications of $G$ have attracted an ever-increasing interest, including the use of $G$ and GRMs for bioelectrodes, bioimaging, drug/gene/peptide delivery, nanopore-based DNA-sequencing, stem cell differentiation and tissue engineering (Feng et al., 2013; Yang et al., 2013). Moreover, GRMs have generated great interests for the design of nanocarriers and nanoimaging tools, two- and three-dimensional tissue scaffolds, anti-bacterial coatings and biosensors (Bitounis et al., 2013; Ding et al., 2015). The interest in using GRMs in medicine lies chiefly upon the extraordinary properties of G, including its mechanical properties, flexibility, transparency, thermo-electrical conductivity and good biocompatibility. GRMs could therefore overcome the limitations of metals and silicon, which are currently used for implantable devices, but are characterized by elevated stiffness, high inflammatory potential and poor long-term stability in physiological environments. Moreover, the biomedical field witnesses a strong need for innovative therapies to assess the increasing demand of more specific, safer and effective treatments for pathological conditions. Given these premises, a large amount of research on $G$ focuses on medical applications, and particularly in the field of neurology, where its mechanical and electronic features make it a strong candidate for replacing current devices (Kostarelos et al., 2017; Reina et al., 2017).

Another appealing aspect of GRM-based medical devices lies on the increasing evidences of $G$ biocompatibility, an extreme important issue to take into consideration for any new biomaterial brought to the market. Due to its chemistry, $\mathrm{G}$ surface allows strong and non-destructive interactions at the cellular level, which could even be improved by specific chemical functionalization (Cheng et al., 2016; Kang et al., 2016). This is particularly true for G-based supports and scaffolds oriented to tissue repair and regeneration, and in fact promising results have already been shown for neural and bone tissue engineering (Cheng et al., 2016; Reina et al., 2017). For what concerns $G$ nanosheet dispersions, mostly intended for drug/gene delivery and diagnostic imaging purposes, the scenario is instead more complex (Bramini et al., 2016; Mendonça et al., 2016a; Rauti et al., 2016). The safety of this material is indeed still a challenging problem to address and every case needs to be analyzed separately by taking into account the synthesis method, the quality of the final product including its purity and the eventual presence of trace contaminants, as well as the biological environment in which $G$ is to be applied.

\section{Graphene Applications in Neuroscience}

The biomedical applications of $\mathrm{G}$ represent a field in continuous expansion. Traditional treatments for central nervous system (CNS) disorders present a number of challenges, thus, developing new tools that outperform the state of the art technologies for imaging, drug delivery, neuronal regeneration and electrical recording and sensing is one of the main goal of modern medicine and neuroscience (Baldrighi et al., 2016). Since the development of carbon-related materials, nanotechnology has strongly impacted a number of applications (Figure 1) including: drug, gene and protein delivery, to cross the blood-brain barrier (BBB) and reach compromised brain areas; neuro-regenerative techniques to restore cell-cell communication upon damage by interfacing two (2D) or three (3D) dimensional scaffolds with neural cells; highly specific and reliable diagnostic tools, for in vivo sensing of disease biomarkers by cell labeling and real-time monitoring of biological active molecules; and neuronal activity monitoring and modulation, by highly sensitive electrodes for recordings and G-based platforms for electrical local stimulation (Mattei and Rehman, 2014; John et al., 2015; Chen et al., 2017; Kostarelos et al., 2017; Reina et al., 2017).

In detail, researchers have already started exploring the use of $\mathrm{G}$ at the CNS for cell labeling and real-time live-cell monitoring (Wang et al., 2014; Zuccaro et al., 2015); delivery to the brain of molecules that are usually rejected by the BBB (Tonelli et al., 2015; Dong et al., 2016); G-based scaffolds for cell culture (Li N. et al., 2013; Menaa et al., 2015; Defterali et al., 2016b); and cell analysis based on G-electrodes (Medina-Sánchez et al., 2012; Li et al., 2015). In addition, interfacing $G$ with neural cells was also proposed to be extremely advantageous for exploring their electrical behavior or facilitating neuronal regeneration by promoting controlled elongation of neuronal processes ( $\mathrm{Li}$ et al., 2011; Tu et al., 2014; Fabbro et al., 2016). These applications open up new research lines in neuro-therapeutics, including neurooncology, neuro-imaging, neuro-regeneration, functional neurosurgery and peripheral nerve surgery (Mattei and Rehman, 2014).

In this review we will focus on few aspects of GRM research that we deem of particular interest for future neuroscience applications, i.e., (i) $G$ as nano-carrier for drug and gene 


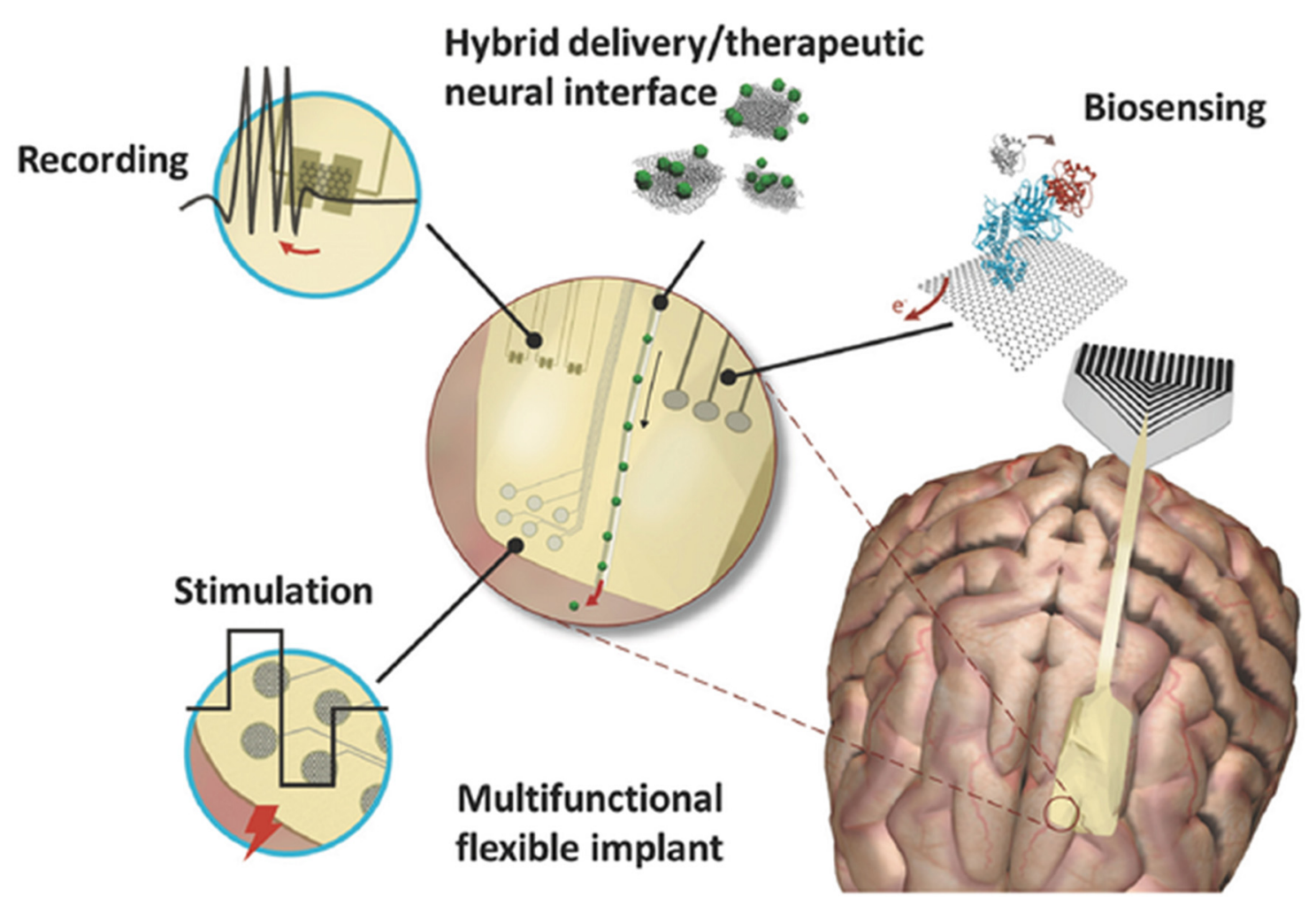

FIGURE 1 | Graphene based neural interfaces for a variety of neuronal functionalities like recording, stimulation and biosensing. Modified with permission from Kostarelos et al. (2017).

delivery; (ii) G interaction with the BBB; and (iii) G-based 2D and $3 \mathrm{D}$ composites for neural regeneration, stimulation and recording. A final (iv) chapter is dedicated to an overview of computational modeling approaches that can help biologists and medical scientists to better understand the molecular and cellular interaction of $\mathrm{G}$ with living systems.

\section{HOW TO REACH THE BRAIN: G-BASED NANOCARRIERS AND THE BLOOD-BRAIN BARRIER}

\section{Graphene Nanosheet Interaction With Neural Cells}

Common mechanisms of cytotoxicity of $\mathrm{G}$ nanosheets have been reported in literature on different cell types, and include the physical interaction with cell membranes (Seabra et al., 2014); disruption of cell cytoskeleton (Tian et al., 2017); oxidative stress due to production of reactive oxygen species (ROS; Chen M. et al., 2016; Mittal et al., 2016); mitochondrial damage (Pelin et al., 2017); DNA damage, such as chromosomal fragmentation, DNA strand breakages, point mutations and oxidative DNA alterations (Akhavan et al., 2012; Fahmi et al., 2017); autophagy (Chen et al., 2014); and apoptosis and/or necrosis (Lim et al., 2016). Furthermore, published data suggest that GO is less toxic than $\mathrm{G}, \mathrm{rGO}$ and hydrogenated-G; smaller nanosheets are less toxic than large flakes and highly dispersible $\mathrm{G}$ solutions are safer than aggregating ones (Donaldson et al., 2006; Akhavan et al., 2012; Bianco, 2013; Kurapati et al., 2016; Ou et al., 2016).

In the case of the CNS, the mechanisms of interaction of GRMs with neurons and astrocytes are still poorly investigated and unclear, depicting an undefined scenario mainly dependent on GRM intrinsic characteristics. Few studies have been carried out in neuronal-like cell lines, showing some toxic effects of $\mathrm{G}$ at high doses. In particular, both $\mathrm{G}$ and carbon nanotubes induced toxic responses in PC12 cells in a concentrationand shape-dependent manner (Zhang et al., 2010). Upon G exposure, ROS were generated and evidences of apoptosis were noticed at a concentration of $10 \mu \mathrm{g} / \mathrm{ml}$. In agreement with this study, GO nanosheets induced no obvious cytotoxicity at low concentration, but dose- and time-dependent cell death was observed in the human neuroblastoma SH-SY5Y cell line (Lv et al., 2012). For what concerns primary cultures, no changes in neuronal and glial cell viability were detected upon $\mathrm{G}$ exposure, both in vivo and in vitro (Bramini et al., 2016; Mendonça et al., 2016b; Rauti et al., 2016). However, primary neuronal cultures exposed to GO nanosheets displayed clear alterations in a number of physiological pathways, such as calcium and lipid homeostasis, synaptic connectivity and plasticity (Bramini et al., 2016; Rauti et al., 2016). Once internalized in cells, G nanosheets were seen to preferentially accumulate in lysosomes, as well as to physically damage mitochondria, endoplasmic 
reticulum and, in some cases, nuclei (John et al., 2015). Another study suggested that the irregular protrusions and sharp edges of the nanosheets could damage the plasma membrane, thus letting $\mathrm{G}$ entering the cell by piercing the phospholipidbilayer (Li Y. et al., 2013). These features raise additional safety concerns, as free GRMs in the cytoplasm may lead to disruption of the cytoskeleton, impaired cell motility and blockade of the cell-cycle, similar to carbon nanotube-induced cytotoxicity.

The above-described effects were observed upon chronic $\mathrm{G}$ exposure, stressing the need of urgent and further biocompatibility assessment of the material with nerve tissues in long-term studies, hopefully linking in vivo effects to in vitro cellular and molecular interactions. A first strong evidence of G-induced CNS toxicity came from a recent in vivo study (Ren et al., 2016). To recreate a situation of G environmental pollution, researchers dispersed GO in water in the presence of Danio rerio (zebrafish) larvae. Exposed larvae displayed GO in the CNS and, most importantly, an induction of Parkinson's disease-like symptoms such as disturbance of locomotor activity, dopaminergic neurons loss and formation of Lewy bodies. These effects were likely a consequence of mitochondrial damage and apoptosis through the caspase 8 pathway, in the presence of a more general metabolic disturbance. $G$ and GO nanosheets accumulate in small quantities in the CNS of rodents after intravenous (i.v.) injection without prior surface functionalization (Mendonça et al., 2016a,b). rGO was also detected in brain tissues, particularly in the thalamus and hippocampus, after i.v. injection that was accompanied by BBB disruption (Mendonça et al., 2016b). Interestingly, rats treated with rGO flakes did not show any clinical signs of neurotoxicity, including no signs of tremor, convulsions, salivation, lacrimation, dyspnea and motor abnormalities. These findings are in contrast with the work carry out by Zhang et al. (2015), who reported the short-term decrease in locomotor activity and neuromuscular coordination in mice orally administered with rGO nanosheets. This discrepancy underlines that the route of administration is key parameter in determining $\mathrm{G}$ biocompatibility. Thus, the portal of entry of $\mathrm{G}$ into the organism, together with its dose, size, functionalization and aggregation, will determine the final biological effects.

In summary, the current data on $G$ nanosheet biocompatibility are still controversial. This is due to the high heterogeneity of materials present on the market and the large variety of synthesis methods. Depending on the graphite source (starting material), the synthesis method, the use of chemicals and the dispersion form (solution or powder) of the final product, $\mathrm{G}$ can present different sizes, thickness, chemical surface and aggregation state, which all affect to various extent its interaction with the biological systems. It is clear, however, that $\mathrm{G}$ nanosheets may cause adverse environmental and health effects, leaving open the debate about their use as biomedical platform (Bramini et al., 2016; Reina et al., 2017). To date, GO nanosheets are preferred with respect to pristine $G$ for biomedical studies, because of their major solubility and stability in biological fluids (Chowdhury et al., 2013; Servant et al., 2014a; Reina et al., 2017).

\section{Graphene for Biomolecule Delivery to the Central Nervous System}

As discussed above, the use of $G$ nanosheet dispersions for biomedical applications could give some unwanted effects due to the intrinsic characteristics of the material. Interestingly, functionalizing the $G$ surface could alleviate most of these drawbacks. The physical-chemical properties of $G$ nanosheets can be tuned toward a higher degree of biocompatibility. Moreover, cargoes can be loaded via $\pi-\pi$ stacking interactions, hydrogen bonding, or hydrophobic interactions (Georgakilas et al., 2016) giving the attractive possibility of using $G$ as a platform for delivery of biomolecules that are usually rejected by the BBB. In fact, the large surface area available and the possibility of conjugating different molecules onto its surface, make $G$ a suitable material for holding and carrying drugs, genes (including siRNA and miRNA), antibodies and proteins (Chen et al., 2013). In addition, it is also possible to modify its chemical structure by adding functional groups such as amino, carboxyl, hydroxyl, alkyl halogen, or azide groups (John et al., 2015). Surface functionalization has the double advantage of loading high quantity of biomolecules and specifically deliver them to target cells, while allowing a more homogenous dispersion of the material, since pure $\mathrm{G}$ is highly hydrophobic and tends to aggregate in aqueous solution, including biological fluids containing salts and proteins (Mattei and Rehman, 2014; John et al., 2015). Additionally, functionalized G nanosheets could be applied in systemic, targeted, and local delivery systems (Feng et al., 2011; Kim et al., 2011; Liu J. et al., 2013). Thus, this approach could fulfill the increasing demand of multifunctional and versatile medical platforms.

Because of its unique fluorescent, photoacustic and magnetic resonance profiles, several studies have also explored the possibility of incorporating G-based nanoparticles (NPs) to enhance the in vivo visualization of brain tumors and improve tumor targeting of molecular anticancer strategies (Kim et al., 2011; Yang et al., 2012; Zhang et al., 2013; Hsieh et al., 2016). Also in this case, in vivo studies revealed that GO, more than GR, has good potential for these applications, in fact, systemically administered radiolabeled GO $\left({ }^{188} \mathrm{Re}-\mathrm{GO}\right)$ could reach the brain parenchyma, although in a small amount $(0.04 \%$; Zhang X. et al., 2011).

\section{Blood-Brain Barrier Crossing}

The $\mathrm{BBB}$ is one of the most important physiological barriers in the organism, forming a dynamic interface that separates the brain from the circulatory system (Pardridge, 2001; Begley, 2004). The barrier is formed by cerebrovascular endothelial cells, surrounded by basal lamina and astrocyte perivascular endfeet that link the barrier system to the neurons (Abbott et al., 2010). Together with pericytes and microglial cells, endothelial cells support the barrier function and regulate its intercellular signaling to control the flow and trafficking to the brain (Dohgu et al., 2005; Abbott et al., 2010). The BBB, together with arachnoid and choroid plexus epithelium, restricts the passage of various chemical substance and foreign materials between the bloodstream and the neuronal tissue, while still allowing 
the passage of substances and nutrients essential to metabolic functions, from oxygen to various proteins, such as insulin and apolipoprotein E (Abbott et al., 2006; Strazielle and GhersiEgea, 2013). An interesting point is that the brain capillary endothelial cells clearly differ from the endothelial cells in the other districts of the body, in that they present a larger number of adherens and tight junctions between adjacent cells, so that no inter-cellular fenestrations exist (Abbott et al., 2006, 2010). The tight junctions between the brain capillary endothelial cells are one of the most important structural and anatomical elements of the BBB. They create the major barrier, associating cell membranes tightly together and regulating paracellular movements of water, molecules, ions and other biomolecules (Begley and Brightman, 2003; Abbott et al., 2010). Based upon these characteristics, some researchers have highlighted that the permeability properties of the $\mathrm{BBB}$ reflect the tightness of intercellular junctions between brain capillary endothelial cells (Rubin et al., 1991). In other words, the low permeability characterizing the $\mathrm{BBB}$ is caused for the most part by tight and adherens junctions that limit paracellular passage (Wolburg and Lippoldt, 2002). The result is that most molecular traffic is forced to take a transcellular route across the $\mathrm{BBB}$, rather than moving paracellularly through the junctions, as in most endothelia (Abbott and Romero, 1996; Wolburg and Lippoldt, 2002; Hawkins et al., 2006).

To date, several mechanisms of transport across the BBB have been identified (Figure 2), including paracellular or transcellular pathways, transport proteins (carriers), receptormediated transcytosis and adsorptive transcytosis (Abbott et al., 2006). Transcytosis is a process whereby biomolecules are engulfed into a plasma membrane invagination and further transported from one side of the polarized cell monolayer to the other side. Specific proteins, such as insulin and transferrin, are taken up by receptor-mediated endocytosis and transcytosis, a process known as receptor-mediated transport (Kreuter et al., 2002; Rip et al., 2009; Ulbrich et al., 2009, 2011). Native plasma proteins, such as albumin, are poorly transported, but cationization can increase their uptake by adsorptive-mediated endocytosis and transcytosis (Abbott and Romero, 1996; Pardridge, 2007a). In addition to transcytosis, very small watersoluble compounds can penetrate the tight junctions through a paracellular aqueous pathway. In paracellular transport, tight junctions act as a "gatekeeper" and regulate paracellular diffusion of water-soluble agents. For example, sucrose is a water-soluble molecule and is able to cross the $\mathrm{BBB}$ in limited amounts by paracellular diffusion (Ek et al., 2006). The large lipid membrane surface area of the endothelium also offers an effective diffusive route (transcellular transport) for small gaseous molecules such as $\mathrm{O}_{2}$ and lipid-soluble agents, including drugs such as barbiturates and ethanol. The endothelium furthermore contains transport proteins for glucose, amino acids, purine bases, nucleosides, choline and other substances. Some transporters, i.e., the P-glycoprotein, are energy-dependent and act as efflux transporters (active-efflux transport).

The complex network of transport systems described above, gives the $\mathrm{BBB}$ a vital neuroprotective function that however comes with some drawbacks, as the $\mathrm{BBB}$ also impedes the passage of drugs for CNS diseases. Pharmaceutical companies have invested significant effort and sums in trying to design drugs that can cross the BBB, with very limited success. It is reported that only $5 \%$ of the total amount of drugs developed for neuronal diseases actually reach the CNS (Pardridge, 2007b).

\section{Nanoparticle Engineering}

The therapeutic potential of NPs exposure depends chiefly on the rate of NP penetration when delivered from the external environment to the internal bio-compartments. Thus, biological barriers are central in determining the biological impact of NP exposure. Nanomaterials offer enormous potential for therapeutics and diagnosis, but also raise the possibility of unintended access to the brain (Herda et al., 2014). In vivo studies showed that NP could be found in the CNS upon various ways of administration (Semmler-Behnke et al., 2008; Zensi et al., $2009,2010)$. In parallel, in vitro models of human and murine $\mathrm{BBB}$ have been used and developed for the investigation of NP translocation (Andrieux and Couvreur, 2009; Ragnaill et al., 2011; Bramini et al., 2014; Herda et al., 2014; Raghnaill et al., 2014).

Numerous nano-delivery systems have been proposed and tested for therapeutic purposes, both in vitro and in vivo (Pandey et al., 2015). Amongst the state-of-the-art systems, polymeric NPs are promising because of their high drug encapsulation capacity, so they protect and transport hydrophobic drugs without damaging the BBB structure (Tosi et al., 2008). Binding apolipoprotein E to NPs has been suggested as a mechanism via which NPs could utilize existing pathways to access the brain (Kreuter et al., 2002; Wagner et al., 2012), and it indeed enhances the uptake of drugs (Michaelis et al., 2006). This approach is particularly promising with liposomes, which are highly biocompatible (Re et al., 2011). In general, exploiting receptor-mediated transcytosis by linking specific peptides to the NP surface has been the most studied system in the field of $\mathrm{BBB}$ crossing. Various molecules, such as transferrin, insulin, lectin and lipoproteins, physiologically use this route to pass from the blood stream to the brain; thus these ligands could increase the passage ratio of drug-loaded NPs through the BBB for therapeutic purposes (Herda et al., 2014; Pandey et al., 2015; Åberg, 2016). Recently, exogenous peptides known to undergo transcytosis in the BBB were also grafted to the NP surface to enhance their entrance in the CNS. Here, particular attention has been given to the diphtheria toxin receptor (DTR) and the Human Immunodeficiency Virus (HIV)-TAT proteins. A mutant of DTR with no toxicity or immunogenicity has been tested to transport nano-liposomes and polybutylcyanoacrylate NPs across the $\mathrm{BBB}$, both in vitro and in vivo, and indeed, only grafted NPs were able to transcytose the barrier (van Rooy et al., 2011; Kuo and Chung, 2012; Kuo and Liu, 2014). The same strategy was successful when using a derivate of the HIV-TAT protein, linked to the surface of polymeric micelles or $\mathrm{SiO}_{2} \mathrm{NPs}$ through polyethylene glycol (PEG) molecules (Liu et al., 2008a,b; Zhao X. et al., 2016). In addition, antibody-grafted NPs have been synthetized to specifically target brain vascular endothelium receptors (Loureiro et al., 2014; Saraiva et al., 2016), in 


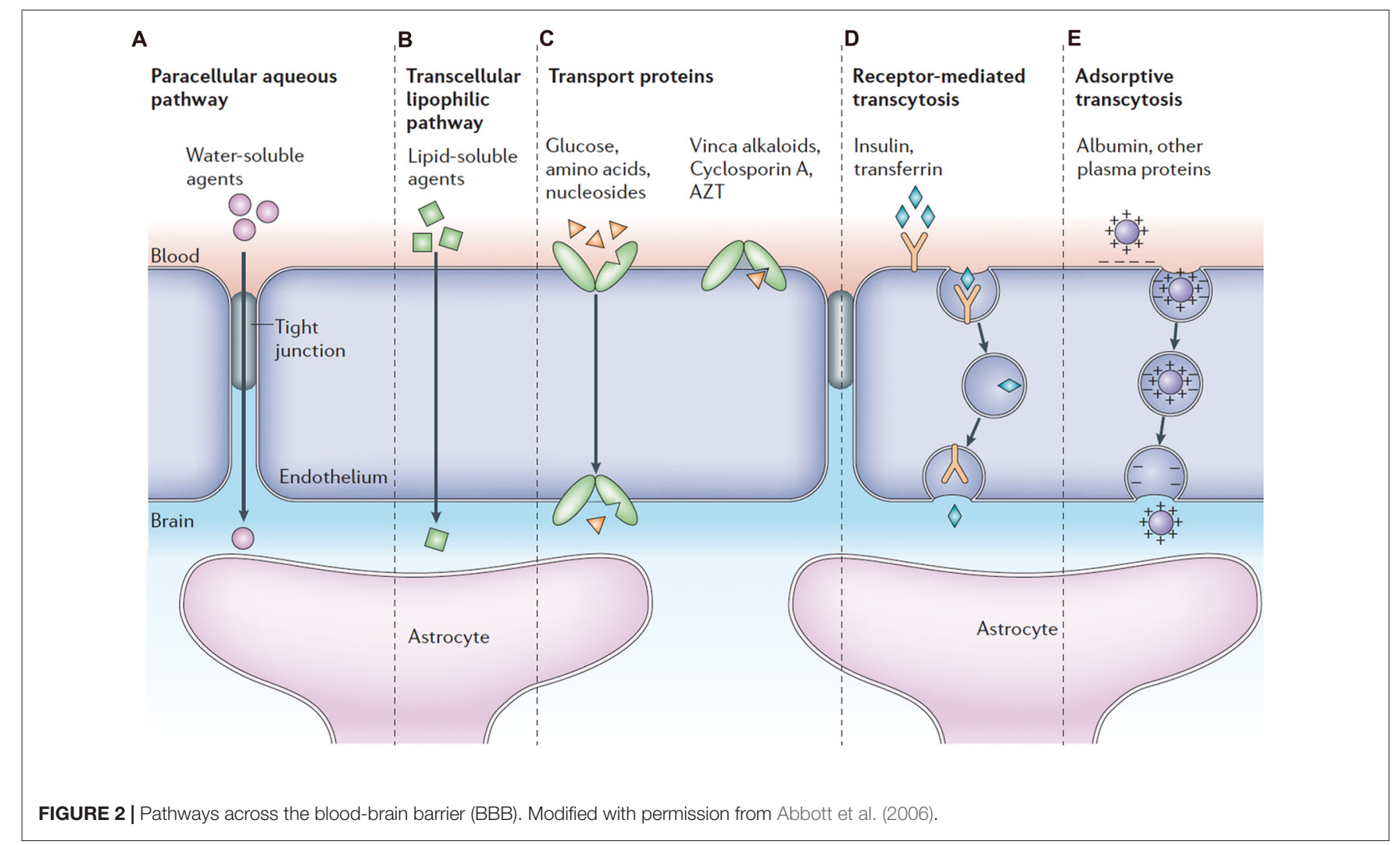

order to exploit the physiological transcytosis mechanisms of the $\mathrm{BBB}$. Once again, the most promising results were obtained with antibodies anti-Insulin (Ulbrich et al., 2011), anti-transferrin (Clark and Davis, 2015) and anti-LDL (Kreuter, 2014) receptors. Even though these recent developments in antibody engineering have improved the knowledge on brain therapeutics, by increasing targeting specificity and avoiding peripheral loss of the material, still significant efforts have to be made to translate these findings from research to clinical applications.

A major challenge of spherical NPs is that it is difficult to obtain a multifactorial engineered system able to encapsulate a drug, cross the $\mathrm{BBB}$ by receptor-mediated endocytosis and finally target a specific cellular subpopulation. In fact, even though NPs present a high surface area, the room for engineering peptides and molecules on the surface to drive and guide the system towards various body compartments is still limited. In this scenario, new approaches that combine external modulation of BBB permeability with NP engineering have been recently developed and are currently under investigation.

\section{Surfactants Coverage and Hyperthermia}

A very similar approach to the above-described NP surface modification with ligands consists in covering NPs with surfactants (Pardridge, 2012). This strategy induces a transient disruption of tight junctions, leading to higher permeability of the endothelium, thus allowing large molecule and nano-carriers to easily cross the BBB and reach the brain (Pardridge, 2012;
Saraiva et al., 2016). Moreover, poly(sorbate 80) can adsorb apolipoprotein E and/or A-I, additionally giving NPs the capacity of binding lipoprotein receptors expressed in the brain endothelium and crossing the BBB (Kreuter et al., 2003; Petri et al., 2007).

In the last few years, researches have adopted innovative strategies with the aim of reduce $\mathrm{BBB}$ damage and increase the amount of drug transported to the CNS. One stream of research aimed at obtaining the time- and area-specific upregulation of BBB permeability, to facilitate NP passage. This was achieved, for example, by activating the A2A adenosine receptor, which increases the intercellular space between the brain capillary endothelium (Gao et al., 2014). A similar effect can be obtained by physically interacting with the BBB by inducing hyperthermia, a procedure that increases the local temperature of the endothelium to $41-43^{\circ} \mathrm{C}$. The change in temperature acts by selectively disrupting tight junctions and increases the paracellular permeability of the BBB. Interesting results were obtained using focused ultrasounds (FUS) and microbubbles, which showed very low tissue toxicity and high accumulation of doxorubicin (DOX) in the CNS (Treat et al., 2007). Other techniques to produce hyperthermia are microwaves and radiofrequency. The latter has been tested in vivo for glioma treatment, in combination with classical chemotherapy and radiotherapy, displaying encouraging results (Wang et al., 2012). Finally, two more advanced strategies to induce hyperthermia have been recently tested: laser pulse and magnetic heating. Near-infrared (NIR) ultrashort 
laser pulses induced BBB disruption in selected regions, thus allowing the passage of large molecules in the brain (Choi et al., 2011). Magnetic NPs (MNPs) were instead used for delivering bioactive compounds via heat generated from magnetic heating, using a low radiofrequency field (Tabatabaei et al., 2015). Since MNP location can also be monitored live, this technique can be applied for both treatment and diagnosis of diseases.

Despite the promising results, techniques that modulate and interfere with the $\mathrm{BBB}$ permeability, even if transiently and locally, are burdened by a major problem, i.e., that there is very poor control over the passage of unwanted molecules and/or microorganisms that populate the blood stream. If it is true that the amount of drug reaching the brain is increased upon tight junction opening, it is also true that toxic compounds, safely constrained to blood vessels by an intact $\mathrm{BBB}$, may pass at the same time, posing high risks to the patient.

\section{Graphene and the BBB: A New Way for Drug and Gene Delivery to the Brain}

The key goal of any drug delivery system is to create a smart tool that recognizes specific targets and releases the drug in a controlled way (Allen and Cullis, 2004). The main limitation of G-based applications in neuroscience is its very low accumulation in the brain parenchyma upon intravenous injection. Once injected intravenously, G will engage with ions, lipids and proteins, resulting in the aggregation of the material and formation of a biomolecular corona that might affect the distribution of $G$ and trigger inflammatory responses (Dell'Orco et al., 2010). In addition, nanosheets can be phagocytosed by macrophages, inducing activation and release of pro-inflammatory cytokines (Zhou et al., 2012), and interact with several blood components inducing hemolysis (Liao et al., 2011). Last but not least, $G$ nanosheets could accumulate in the reticulo-endothelial system rather than in the tissue to which they are targeted (McCallion et al., 2016).

Particularly challenging is the passage through the $\mathrm{BBB}$, which significantly limits the delivery of drugs, blocking roughly the $100 \%$ of large molecule neuro-therapeutics and more than $98 \%$ of all small-molecule drugs (Upadhyay, 2014). Accordingly to Mendonça et al. (2016b) systemically injected rGO nanosheets cross the $\mathrm{BBB}$ through a transitory decrease in the $\mathrm{BBB}$ paracellular tightness and accumulate in the thalamus and hippocampus of rats. On the contrary, functionalization of rGO with PEG, usually used to improve biocompatibility of nanomaterials, induces BBB breakdown and astrocyte dysfunctions in vivo (Mendonça et al., 2016a). Among the various approaches to make $\mathrm{G}$ cross the $\mathrm{BBB}$, ultrasounds were applied to mice to physically open $\mathrm{BBB}$ tight junctions and allow the drug delivery system to enter the brain. By following this method, GO nanosheets grafted with Gd-DTPA and poly(amidoamine) dendrimer, and loaded with EPI and the tumor suppressor miRNA Let-7, could reach the brain upon tail vein injection (Yang et al., 2014). The main advantage of this approach is the reversibility of the $\mathrm{BBB}$ opening. Interestingly, $\mathrm{G}$ allowed at the same time high contrast MRI analysis and a quantification of the distribution of the delivery system inside the brain tissue (Yang et al., 2014). These results are promising, however in-depth pharmacokinetics and toxicological studies are needed, especially for long-term treatments, keeping in mind that, with respect to what has been studied so far, this technique achieves a much higher $G$ accumulation in the CNS.

Alternatively, G surface can be functionalized with specific biomolecules that enable the material to cross the BBB (Allen and Cullis, 2004; Goenka et al., 2014; John et al., 2015). A recent study has investigated an innovative nano-delivery system with high loading capacity and a $\mathrm{pH}$ dependent behavior. The $\mathrm{GO} @ \mathrm{Fe}_{3} \mathrm{O}_{4}$ nanocomposite was conjugated to lactoferrin (Lf), an iron transporting serum glycoprotein that binds to receptors overexpressed at the surface of vascular endothelial cells of the $\mathrm{BBB}$ and of glioma cells, in order to obtain Lf@GO@ $\mathrm{Fe}_{3} \mathrm{O}_{4}$. After loading the NPs with DOX, a drug used to treat glioma (Figure 3), NPs were intravenously injected and the particles were seen to migrate from the bloodstream to glioma cells (Liu G. et al., 2013). NPs were more concentrated in the CNS compared to other organs, and a higher efficiency in tumor regression was observed, compared to the control of animals injected with DOX alone. Following a similar approach and with similar promising results, Yang L. et al. (2015) functionalized PEG-GO nanosheets with the Tat protein of the HIV, which allowed the drug-loaded PEG-GO system to cross the BBB by transcytosis, while leaving the barrier endothelium fully preserved.

As previously discussed, another promising strategy to challenge the BBB is NP coating with surfactants (Kreuter et al., 2003; Gelperina et al., 2010). Kanakia et al. (2014) improved GO delivery to the CNS by functionalizing the nanosheets with dextran; the material was found to cross the $\mathrm{BBB}$ and reach the brain without exerting toxic effects. Surprisingly, the GO concentration in the CNS increased with time, while remaining almost absent in other organs. Thus, the study suggests a slow accumulation of $\mathrm{G}$ in the CNS and long-term persistency of the material, that is encouraging from the point of view of the drug delivery system, but also raises safety concern on long-term toxicity of $\mathrm{G}$ nanosheets (Baldrighi et al., 2016), an issue that still needs to be assessed.

The number of drugs successfully linked to $G$ nanosheets is increasing. Liu Z. et al. (2008) showed that GO-PEG flakes could be decorated with the water insoluble aromatic molecule 7-ethyl-10-hydroxy-camptothecin (SN38), via non-covalent van der Waals interactions. Similarly, other drugs, including different camptothecin analogs (Liu Z. et al., 2008), Iressa (gefitinib; Liu Z. et al., 2008), and DOX (Sun et al., 2008), were successfully attached onto the GO-PEG complex by simple non-covalent binding. rGO-PEG particles were able to cross the endothelial layer of the $\mathrm{BBB}$ without disrupting the tight junctions, in both in vitro and in vivo studies (Mendonça et al., 2016a,b). Recently, Xiao et al. (2016) used GQDs conjugated to a neuro-protective peptide. Once injected intravenously in a murine model of Alzheimer disease, they were able to increase learning and memory, dendritic spines formation and decrease pro-inflammatory cytokine levels. 


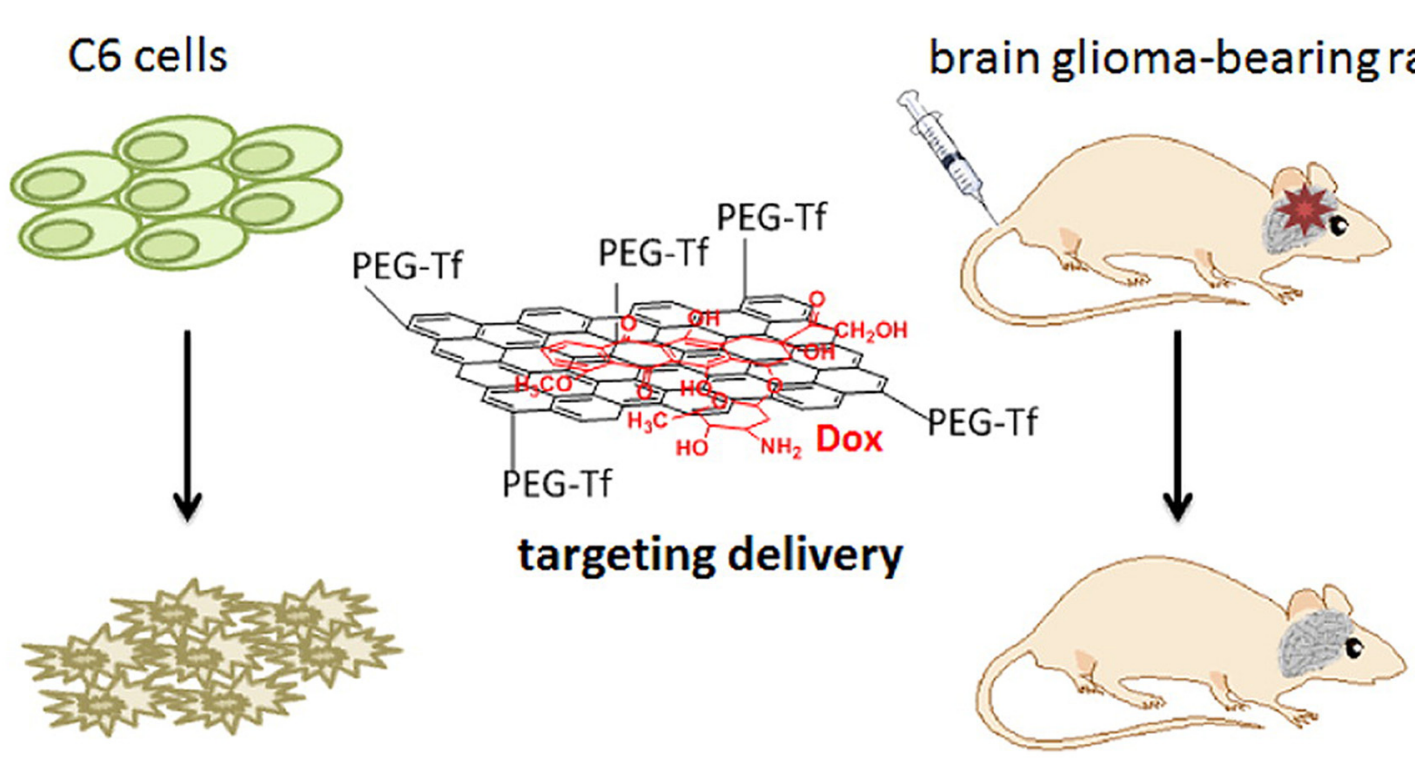

FIGURE 3 | Transferrin modified G oxide (GO) for glioma-targeted drug delivery. Modified with permission from Liu G. et al. (2013).

One of the main applications of G-based drug delivery systems is anticancer therapy, by linking $\mathrm{G}$ composites with chemotherapeutics. Given their strong optical absorbance in the NIR region, G-based hybrid materials are also intensively studied for their promising applications in cancer phototherapy (Liu et al., 2011; Robinson et al., 2011; Yang et al., 2012; Hönigsmann, 2013). The rationale beyond this approach is to exploit the heat produced by the $\mathrm{G}$ accumulated in tumor regions upon NIR laser stimulation to kill cancer cells. This technique was successfully applied in vitro using U251 glioma cells (Markovic et al., 2011). Such experimental approaches are of special interest, as they might help overcoming the limitations imposed by the BBB (Abbott, 2013), and are very promising especially for the treatment of very resistant and aggressive tumors, such as the glioblastoma.

The intrinsic properties of $\mathrm{G}$ in the visible (VIS) and NIR range make it also an attractive tool for bio-imaging (Zhang et al., 2013) both in vitro and in vivo (Gollavelli and Ling, 2012). For example, aptamer-carboxyfluorescein/GO complexes were employed for intracellular monitoring and in situ molecular probing of specific clusters of living cells, such as artificially implanted tumors in mice. GO nanosheets were also used for photo-acoustic imaging, which relies on the acoustic response on heat expansion following optical energy absorption (Wang et al., 2010; Yang et al., 2010; Qian et al., 2012). Specifically for CNS applications, in vivo studies showed that intracranial administered PEG-GO and its derivatives can be imaged in the brain by two-photon microscopy (Qian et al., 2012). Through this imaging technique, a 3D distribution map can be reconstructed in the brain parenchyma due to the high tissue penetration of the fluorescence signal of PEG-GO composites. These promising results could lead to the use of $G$ as a diagnostic tool for imaging brain cancerous lesions, especially if the material is engineered with biomolecules that specifically target tumorigenic cells. Furthermore, once the targeting is achieved, G properties can be optimized according to the specific application, i.e., the size and oxidation state might be changed to shift the emission wavelength from VIS to NIR, which has a deeper tissue penetration, thus improving the depth of the diagnostic imaging device. By combining the optical properties of $G$ with other biodegradable and functional materials, it will be possible to create G-based composites and hybrids suitable for several live-imaging applications. So far, most of the tools have been tested in vitro on cancer cell lines, and in vivo for cancer detection and diagnosis, leaving unaddressed the possibility of using them to explore and image the CNS (Zhang et al., 2013; Cheng et al., 2016).

Similarly to drug delivery, also genetic engineering can exploit $\mathrm{G}$ properties and open new opportunities in biomedicine. The concept in this case would be to deliver nucleic acids, i.e., DNA or various types of RNA molecules, including miRNA and shRNA, to specific target cell populations, to restore physiological conditions (Cheng et al., 2016). The development of non-viral systems is of great importance for future medical approaches as $G$ could allow overcoming some of the intrinsic limitations of viral systems, such as difficulties in accommodating long nucleic acids, batch-to-batch variations, elevated costs and the immunogenicity of viral vector systems (Kim et al., 2011; John et al., 2015). Different strategies have been developed, including the decoration with positively charged polymers (PEI, BPEI), dendrimers (PAMAM) and polysaccharides, which enhance gene transfection efficiency by promoting the interaction with the cell membrane (Liu et al., 2014; Paul et al., 2014). Being the technique of functionalization the same, both drugs and 
genes can be delivered simultaneously using G-based hybrid materials (Zhang L. et al., 2011). This would exhibit a synergic effect, as it would bring a significant enhancement of drug as well as transfection efficiency. On this line, G-nanosheets were functionalized with the cationic polymer PEI, a non-viral gene vector that forms strong electrostatic interactions with the negatively charged phosphate groups of both RNA and DNA (Feng et al., 2011). A step further was taken by Chen et al. (2011) that used PEI-functionalized GO for gene delivery yielding a high transfection efficiency in the absence of any cytotoxic effect.

In summary, G-based delivery systems, when conveniently functionalized or associated with complementary technologies, represent promising candidates for both diagnostics (i.e., imaging) and therapeutics (i.e., drug and gene delivery) neuroscience applications. Moreover, in spite of few studies showing toxic effects of exposure the nervous system to bare G and rGO (Bramini et al., 2016; Mendonça et al., 2016b; Rauti et al., 2016), to date there is no solid evidence that functionalized-G is harmful to neuronal cells and the BBB. Since G-based technologies for biomedical applications are constantly and rapidly evolving, the near future may see the development of new safe and highly neurocompatible materials.

\section{GRAPHENE SUBSTRATES AS NEURONAL INTERFACES}

Tissue engineering aims to restore the functionality of a disrupted tissue by interfacing it with suitable biomaterials. This is a fast-expanding field of research in need of innovative approaches to achieve highly biocompatibile, functional and low invasive implants for long-term applications. For what concerns the nervous system, active and dynamic implantable devices are extremely advantageous as they allow to simultaneously stimulate and record electrical activity of neural cells. Various types of implantable devices have been developed to be used as neural interfaces. Amongst these are deep brain stimulations implants (DBI) for the electrical stimulation of deep structures in the CNS, clinically used to treat dystonia and tremor in Parkinson's disease (Perlmutter and Mink, 2006), retinal and cochlear implants to electrically stimulate the surviving neurons in the presence of retinal degeneration or to convert external sounds in electrical impulses (Spelman, 2006; Picaud and Sahel, 2014), central and peripheral nervous system stimulators for motor rehabilitation after spinal cord lesions (Hatsopoulos and Donoghue, 2009), and intracranial electrodes to map brain electrical activity for diagnostic purposes (Chang, 2015).

The intrinsic properties of $G$ can be exploited to design G-based devices for neuronal interfaces, as $G$ can enhance the optical, electrical and mechanical properties of composite nanostructures. In general, fundamental requirements for a good neural implant are a good biocompatibility coupled to minimal inflammatory response, adequate signal-to-noise ratio if neural recordings are envisaged, and minimal invasiveness, preserving the integrity of the implanted tissue. Typically, G-based scaffolds can be classified according to their dimensionality, i.e., one-dimensional (fibers, ribbons or yarns), two-dimensional (papers, films) and three-dimensional (Cheng et al., 2016; Reina et al., 2017). The most common applications of G-based structures in nanomedicine include the engineering of scaffolds for in vivo neuronal regeneration, stimulation and recording, and for on-demand drug delivery (Cong et al., 2014; Cheng et al., 2016). For what concerns in vivo applications, the use of $2 \mathrm{D}$ devices is mostly limited to planar electrodes (Liu et al., 2016; Park et al., 2018). In fact, several G-based 2D devices have been engineered, but due to technical limitations, so far they have been tested mainly in vitro (for a comprehensive review of $2 \mathrm{D}$ G-based substrates applied to neuronal cells see (Bramini et al., 2018). Promising in vitro results have been recently obtained by Defterali et al. (2016a). Uncoated thermally reduced graphene (TRG) substrates were used to grow and differentiate neural stem cells (NSCs), which grew directly on the G material without any prior biomolecule coating. Cultures grown on TRG substrates were characterized by higher cell number and more synaptic boutons, as well as by efficient multi-lineage differentiation, compared with cultures grown on carbon nanotube-substrates, suggesting a potential use of $G$ to study functional neuronal networks. In the case of CVD-G, instead, the limiting step is the transfer of the monolayer $G$ onto the final substrate, a process that often creates contaminants and defects in the G structure. In addition, a suitable substrate that will interfere as less as possible with the chemical-physical characteristics of $\mathrm{G}$ is still to be found. Furthermore, 2D devices were less active in vitro with neuronal stem cells compared to $3 \mathrm{D}$ scaffolds with the same surface chemistry (Jiang et al., 2016), clearly indicating that morphology, dimensionality, accessibility and porosity are critical scaffold features. Indeed, foams and hydrogels are the scaffolds of choice to drive regeneration in the brain, while directional conduits are preferred to drive re-growth of peripheral nerves. In the next paragraph we will discuss the latest developments in the use of 3D G-based scaffold in neuroscience, focusing on the link between the $\mathrm{G}$ content and structure of the device, and its functionality.

\section{D G-Based Scaffolds: Composites, Foams, Fibers and Hydrogels}

Applications of G-based materials in the neurology field will only be possible upon development of three-dimensional scaffolds able to support nerve regeneration across the injured/lesioned site. The unique properties of planar 2D G-scaffolds are exceeded by $3 \mathrm{D}$ G-structures, which provide a microenvironment where cells are able to grow under conditions that are closer to the in vivo situation. In addition, as previously mentioned, 3D structures possess an enormous interface area and provide highly conductive pathways for charge transport, useful to support neural network formation and neuronal regeneration.

Several 3D scaffolds have been generated and tested in vitro, however so far only a very limited number of them have also been implanted in vivo (Figure 4). Some examples include G-coated electrospun PCL microfiber scaffolds, which were implanted in the striatum or the subventricular zone of adult rats. G-coated implants were associated with a lower microglia/macrophage 
infiltration when compared to bare scaffolds, while supporting astrocytes and neuroblast migration from the SVZ (Zhou et al., 2016). Free-standing 3D GO porous scaffolds were implanted in the injured rat spinal cord, showing no local or systemic toxicity and a good biocompatibility also in the case of chronic implantation (López-Dolado et al., 2015). Of note, long-term (30 days) implants were able to promote angiogenesis and partial axonal regeneration (López-Dolado et al., 2016). No attempts have been made so far to use G-based materials to drive peripheral nervous system regeneration. A first step in this direction is represented by the engineering of G-silk fibroin composite nanofiber membranes. This composite material is of interest as it combines the electrical conductivity and mechanical strength of $\mathrm{G}$ with the good compatibility of silk. Although they have not been tested in vivo, G-silk membranes support the growth of Schwann cells in vitro (Zhao et al., 2017).

Various three-dimensional G and GO foams were shown to be compatible substrates for stem cells (Crowder et al., 2013; Li N. et al., 2013; Serrano et al., 2014; Guo et al., 2016; Sayyar et al., 2016). Li N. et al. (2013) firstly described 3D G-based foams (3D-GFs) as suitable scaffold for NSCs growth and proliferation. NSCs grown on 3D-GFs were able to differentiate into neurons and astrocytes; moreover, it was also noticed that 3D-GFs were optimal platforms for electrical stimulation of NSCs in order to enhance their differentiation. Similar results have been obtained more recently with rGO microfibers, which could support NSC viability and drive them toward a neuronal phenotype (Guo et al., 2017). Interestingly, the features of the $G$ scaffolds (i.e., stiff vs. soft) differentially affected cell adhesion and proliferation and could drive NSC differentiation toward the astrocyte and neuronal lineages, respectively ( $\mathrm{Ma}$ et al., 2016). Hippocampal neurons cultured on 3D-GFs are characterized by a more extensive connectivity associated to a higher network synchronization with respect to $2 \mathrm{D}-\mathrm{G}$ substrates, thus better mimicking the physiological properties of the brain (Ulloa Severino et al., 2016). Microglial cells were also grown on $3 \mathrm{D} G$ foams. In this case, the $3 \mathrm{D}$ structure of the scaffolds affected the neuroinflammatory response of the cultured cells, probably because of spatial constraints due to the 3D topographic features (Song et al., 2014). Similar to what described for 2D materials, also 3D G/GO scaffolds were used as cell stimulating electrodes, to drive neuronal growth and differentiation of NSCs (Li N. et al., 2013; Akhavan et al., 2016).

A new generation of electro responsive $3 \mathrm{D}-\mathrm{G}$ scaffolds is also being developed, i.e., G-based hydrogels, which mimic soft tissue and have been proposed for controlled, stimulationtriggered drug release applications. Hybrid G-based hydrogels are synthetized mainly using GO, G oxide peroxide (GOP) or rGO, by incorporating very low amounts of the material into a hydrogel matrix, to enhance its electrical, mechanical and thermal properties (Servant et al., 2014b). Such materials are able to support neuronal growth and the development of synaptic activity (Martín et al., 2017). Following a similar approach, dexamethasone, a corticosteroid medication, was loaded onto poly(lactic-co-glycolic) acid NPs that were subsequently added into alginate hydrogels. The final composite was used as coating of gold and iridium electrodes for local drug administration after implantation (Kim et al., 2004; Kim and Martin, 2006). These or similar strategies could be employed to engineer smart coating for neuronal implants, with the final goal of having a device able to release biologically active molecules upon controlled electrical stimuli, at the same time improving the surface softness and enhancing the biocompatibility of the implants.

Altogether, the use of $\mathrm{G}$ materials in 3D implants aimed at neuroscience applications is still limited. However, much is to be learnt from other fields of biomedicine. For example, G-hydrogels and foams have recently been proposed for anticancer therapy (Xu et al., 2017; Zhang et al., 2017), as well as for guided bone (Lu et al., 2016), cartilage (Nieto et al., 2015) and muscle (Mahmoudifard et al., 2016) regeneration. We expect that the cross-fertilization between these different disciplines will lead in the close future to the development of functional 3D, G-based implants for nervous system applications.

\section{Graphene-Based Devices for Neural Recording and Stimulation}

Clinical interventions for the recovery of neural dysfunctions and motor disorders attract and challenge the research toward implantable stimulation devices able to adapt to flexible supports and possibly outperform the most common metal electrodebased technologies. Polymeric interfaces outperform in terms of mechanically compliant properties, but often lack durability under physiological conditions, and, above all, proper electrical conductivity. Most of the neural stimulation performed so far with G-based electrodes in contact with living neuronal tissues or cells has been limited to modulate their growth and/or differentiation (Thompson et al., 2015).

Neural stimulation techniques, such as deep brain or cortical stimulation, cochlear and retinal implants, usually rely on the ability of the implanted devices to elicit a functional response of the tissue by providing minimum injected charge, and therefore require electrodes (Kostarelos et al., 2017). To date, in vivo studies show that $\mathrm{G}$ electrodes can stimulate and record neuronal activity. G electrodes produce slightly higher values of charge injection with respect to common noble metal electrodes, like Pt or Au. New promising materials and compounds exploit G to reach up to tens of $\mathrm{mC} \mathrm{cm}^{-2}$ charge injection levels, like in the case of an in vivo probe of laser reduced GO embedded into parylene-C (Apollo et al., 2015). The authors employ the novel flexible freestanding electrodes both to stimulate retinal ganglion cells ex vivo as much as to record neural activity in vivo from cat visual cortex. This constitutes one of the few reported evidences of neural stimulation with a G-based device. Other interesting applications make use of copper microwires encapsulated with CVD-G for an MRI compatible neural device (Zhao Y. et al., 2016), or flexible G micro-transistors for the mapping of brain activity (Blaschke et al., 2016) just to mention a few, but still limited to the recording of neural activity in vivo. In addition, Kuzum et al. (2014) also developed a flexible, low noise G electrode for simultaneous electrophysiology and imaging recording in vivo. 
A
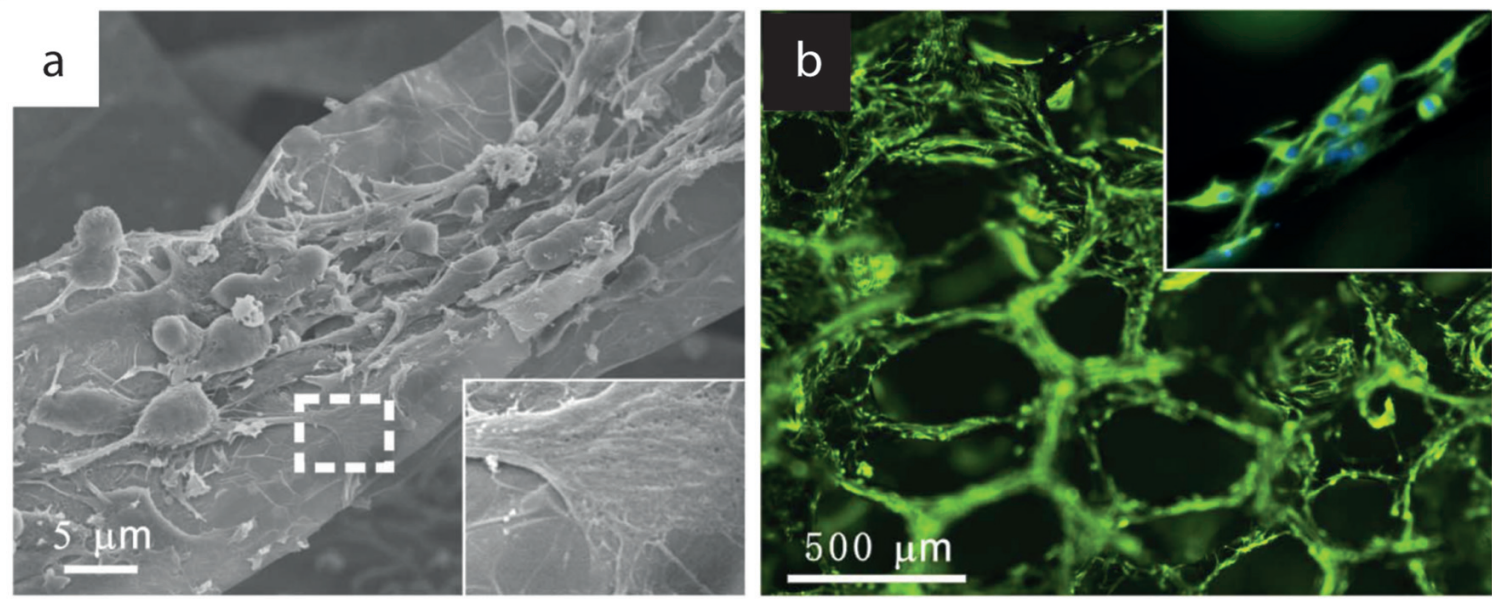

B
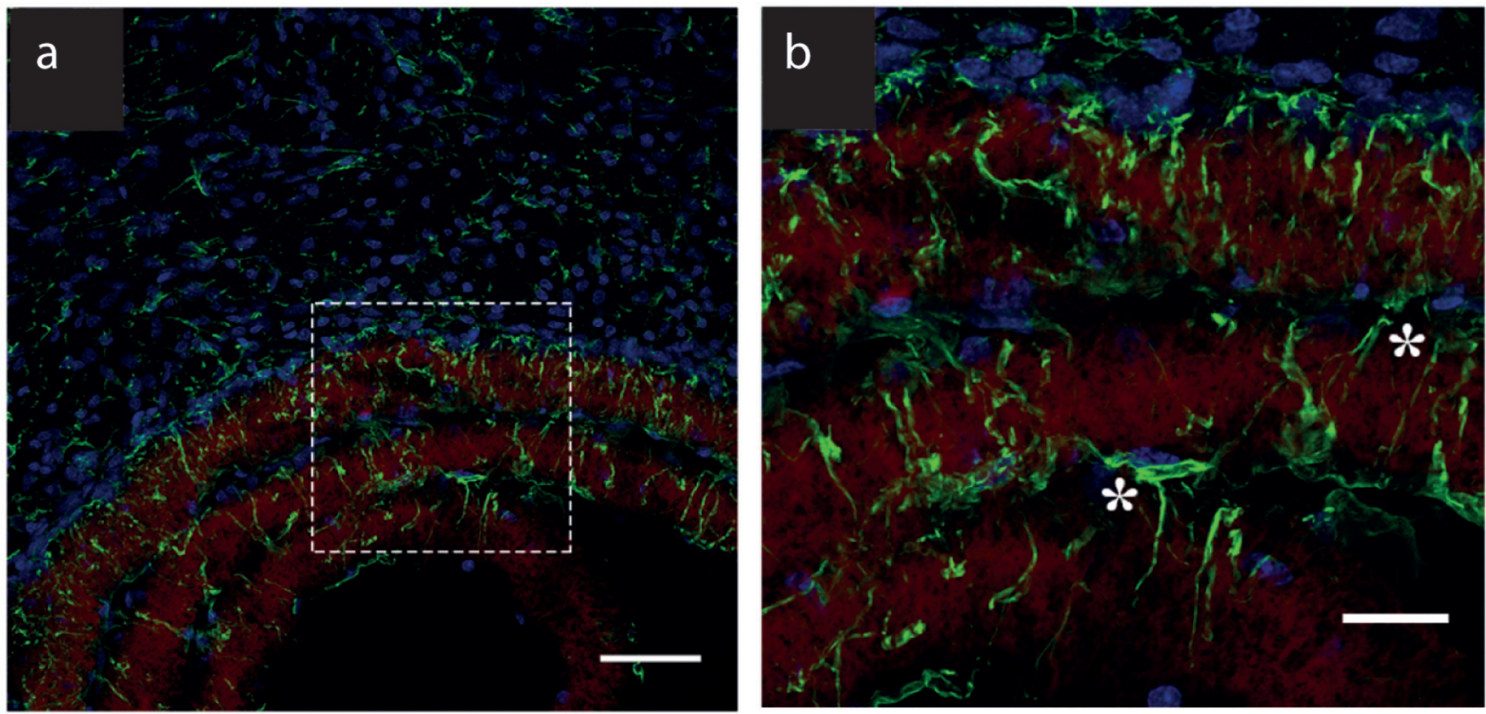

FIGURE 4 | 3D G-Scaffolds in vitro and in vivo. (A) (a) SEM images of neural stem cells (NSCs) cultured on 3D-G foams under proliferation conditions. The insets illustrate the interaction between the cell filopodia and surface. (b) Fluorescence images of NSCs cultured on 3D-G foams for 5 days. Nestin (green) is a marker for NSCs, and DAPI (blue) identifies nuclei. Modified with permission from Li N. et al. (2013). (B) (a,b) Brain astrocyte/G-scaffolds interaction and astrocyte process infiltration 3 weeks after scaffold implantation. Green: GFAP-positive astrocytes, blue: DAPI-stained nucleus, red: surface-functionalized scaffolds. (b) Detailed astrocyte morphology of the dash-box indicated area in (a). ${ }^{*}$ Indicate astrocytes that bridge a gap between two scaffold layers. Scale bar, 50 (a) and 20 (b) $\mu \mathrm{m}$. Modified with permission from Zhou et al. (2016).

After bicuculline injection to evoke epileptiform activity, it was possible to register simultaneously from rat cortical hemispheres with $\mathrm{G}$ electrodes and $\mathrm{Au}$ electrodes of the same size. The $\mathrm{G}$ electrodes showed six times lower signal-to-noise ratio with respect to $\mathrm{Au}$ electrodes, suggesting that the adoption of the new G-based recording system could offer clear advantages for studying brain electrical activity (Figure 5). In addition, thanks to the transparency of the $G$ electrodes, it was also possible to image the cortical area, combining in vivo two-photon imaging and cortical electrophysiological recording (Kuzum et al., 2014).

A further progress has been achieved by developing $G$ fieldeffect transistors (G-FETs), which allow signal amplification reducing external noise (Veliev et al., 2017). Flexible G-based supercapacitors showed recently their potential for neural stimulation thanks to their improved double layer capacitance when hybridized with polymeric materials, like PEDOT:PSS and rGO, G-polyaniline nanocomposites or CVD GO foams (Yang W. et al., 2015; Hu et al., 2016). Another way to exploit $G$ in bio-medical applications has been to enhance the optoelectronic properties of photosensitive neural interfaces deputed to the recovery of compromised vision. A polyimide array of photodetectors based on $\mathrm{MoS}_{2}$ and inkjet $\mathrm{G}$ has recently been proposed as flexible retinal prosthesis, and tested for biocompatibility in vitro (Hossain et al., 2017). Nevertheless, most of these efforts need yet to be translated into usable electrodes for neuroscience applications. 
A a

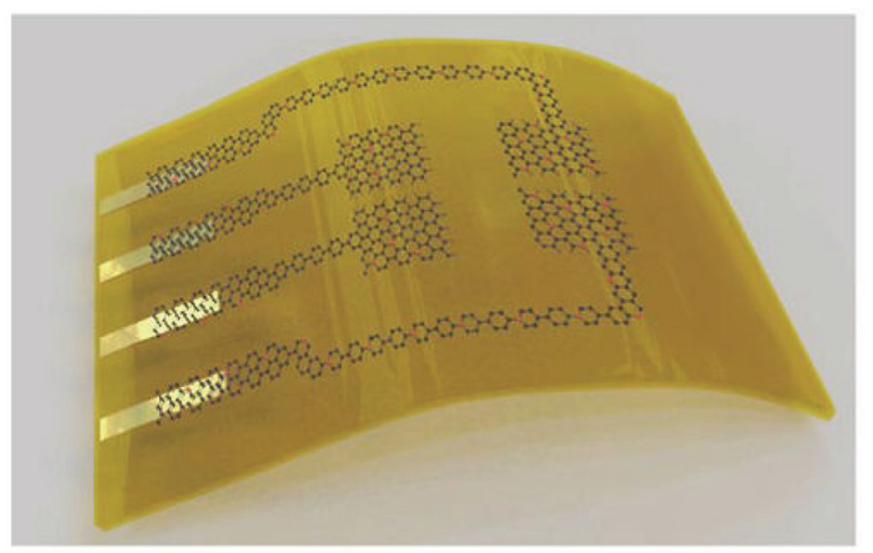

b

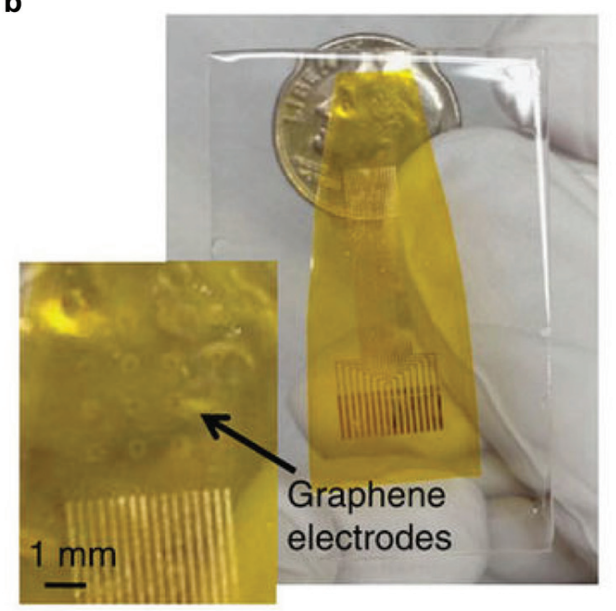

B

a

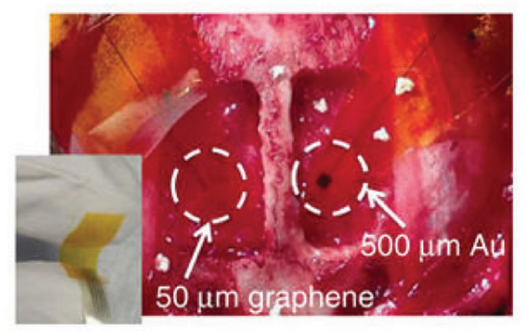

b

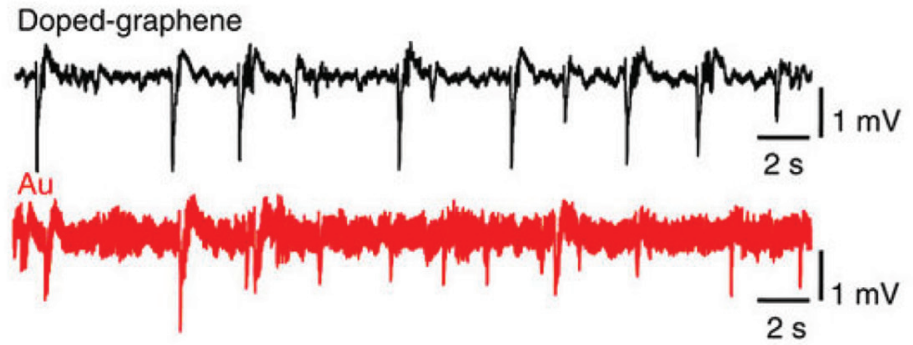

FIGURE 5 | Graphene electrodes for in vivo recording. (A) (a) Schematic illustration of a flexible G neural electrode array. (b) Photograph of a 16-electrode transparent array. The electrode size is $300 \times 300 \mu \mathrm{m}^{2}$. (B) (a) Photograph of a $50 \times 50 \mu \mathrm{m}^{2}$ single-G electrode placed on the cortical surface of the left hemisphere and a $500 \times 500 \mu \mathrm{m}^{2}$ single-Au electrode placed on the cortical surface of the right hemisphere. (b) Interictal-like spiking activity recorded by $50 \times 50 \mu \mathrm{m}^{2}$ doped-G and Au electrodes. Recordings with doped-G electrodes are five- to sixfold less noisy compared with the ones with same size Au electrode. Modified with permission from Kuzum et al. (2014).

\section{COMPUTATIONAL MODELING AND SIMULATIONS OF GRAPHENE-INTERACTING BIOMOLECULAR SYSTEMS}

Understanding the fine structural details underlying interactions between biomolecules and inorganic surfaces is pivotal for many applications in nanomedicine. Although relevant experimental results about the dynamics of these interactions have been recently reported, many topological details remain unclear, especially at the initial events at ns to $\mu$ s timescales. To fill this gap, the use of computational modeling and Molecular Dynamics (MD) simulations gives a relevant contribution, providing details that cannot be accessed by experimental techniques (Ozboyaci et al., 2016).

With its promising properties, $G$ has shown great potential in various applications, and the number of computational studies devoted to it is in constant growth (Cavallucci et al., 2016). Classical MD simulations (i.e., based on a classical physics description of atom-atom interactions) produced in the recent years a large amount of results on the interaction between G-based materials and biomolecules. In particular, these studies allowed to deeply characterize $G$ as a substrate or nanopore for the deposition of biomolecules, differentiating the behavior of pristine $\mathrm{G}$ from that of GO. Moreover, MD simulations have been widely used to test $G$ biocompatibility by studying its interaction with different biological structures such as membranes and protein complexes. In these studies, $G$ has been described as a promising vector against bacterial agents as well as a material capable of perturbing biological complexes.

The most important problem in classical simulations of these systems, still now under debate, is the definition of an appropriate set of force fields parameters for $G$, to allow the implementation of successful simulations with mainstream software packages used for the simulations of biological systems, e.g., GROMACS, (Abraham et al., 2015), CHARMM (Brooks et al., 2009) or NAMD (Phillips et al., 2005). Although different choices have been investigated, it is commonly accepted to describe $\mathrm{G}$ atoms as uncharged Lennard-Jones spheres (Hummer et al., 2001; Patra et al., 2009, 2011). A list of G parameters used in different force fields has been recently reported 
A

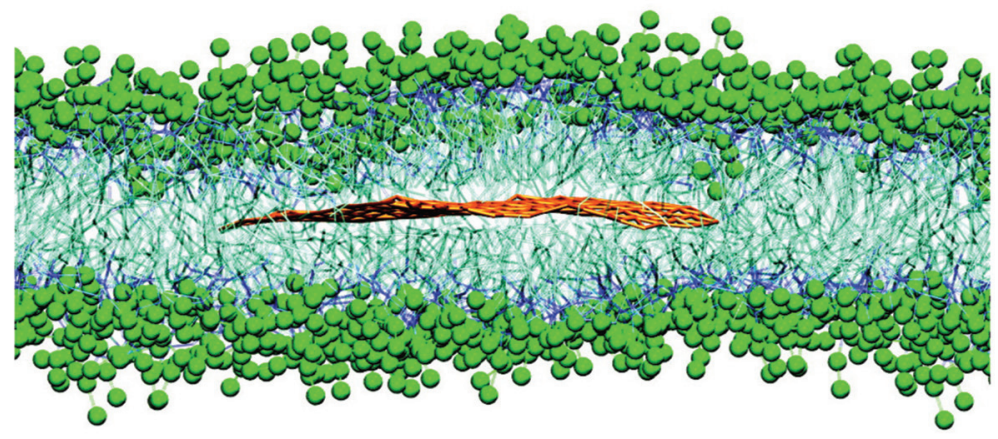

B

a

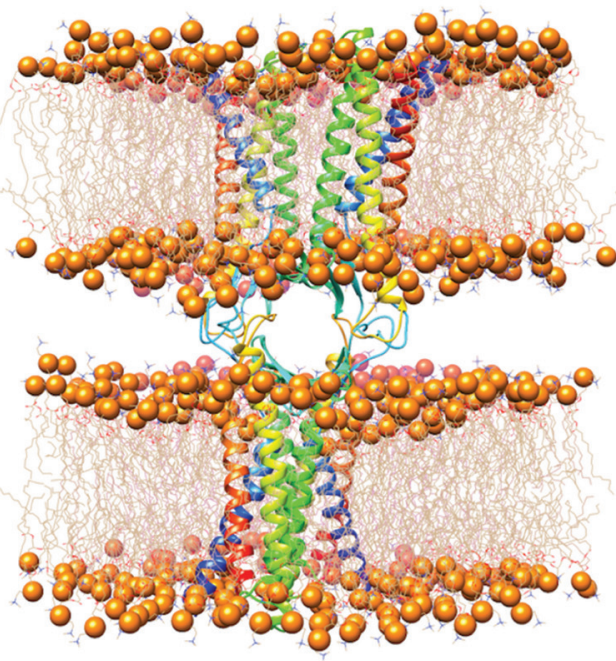

b

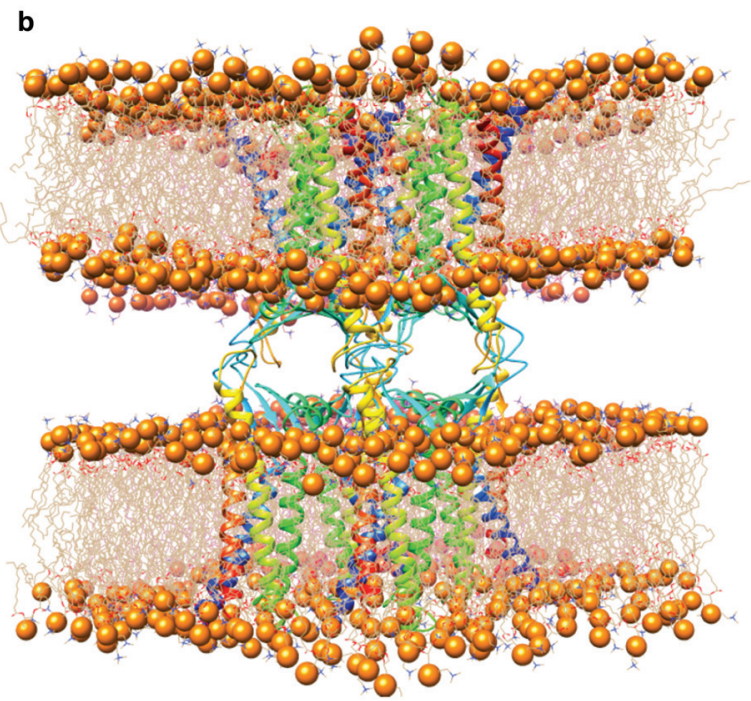

FIGURE 6 | Graphene interaction with biomembranes. (A) Equilibrated superstructure of a graphene sheet inside the phospholipid bilayer formed by 1-palmitoyl-2-oleoyl-sn-glycero-3-phosphocholine (POPC) lipids. Polar heads of the POPC lipids are depicted as green beads, hydrophobic hydrocarbon chains as thick blue lines; the graphene sheet is shown with brown lines (water molecules not shown; modified with permission from Titov et al., 2010). (B) The structure of single (a) and double (b) Cldn15-based paracellular pores, after the respective equilibration protocols. Protomers are shown as ribbons. Each cis dimer is embedded in a hexagonal POPC bilayer, shown as wire structures with phosphorus atoms as spheres. Modified with permission from Alberini et al. (2017).

(Pykal et al., 2016). The aim of this paragraph is to summarize the major findings of computational studies on the interactions between G-based materials and biomolecules, broadly studied at a multi-scale level. A variety of different approaches have been used, such as all-atom or coarse-grained models, and different parameters for interaction studies (the so-called force fields).

A general problem in computational biophysics is the gap between the size and time-scale that can be investigated by simulations and those of biologically relevant mechanisms. Molecular modeling is able to describe biological systems with all-atom details, but this limits its application to study systems of at most $\sim 150 \mathrm{~nm}$ and on the microsecond time-scale. A possible way to bridge this gap is to use coarse-grained molecular dynamics (CGMD) simulations, which are based on a controlled reduction of the number of degrees of freedom and the use of shorter-range interacting functions. Due to these simplifications, a CG simulation has a minor resolution but requires less computational resources, allowing the study of larger systems for longer time-scales. A promising approach is to employ a multiscale description, by alternating the use of classical all-atom with CGMD simulations.

Several computational studies about G-biomolecules systems have been recently published, which can be grouped in the following thematic areas:

1. The adsorption of proteins and peptides (with a particular interest for enzymes and blood proteins) on $G$ substrates, in the context of the study of functional architectures for biomedical applications. Results show that GO has a good solubility in aqueous solution and other organic solvents, thanks to the oxygen-containing groups which can act as reaction sites for the binding of different molecules. As an example, the immobilization of enzymes on a solid substrate is an efficient process to improve its activity while a major factor determining the biocompatibility of a nanomaterial in contact with blood, e.g., medical implants, is the adsorption of proteins on its surface. 
2. The interaction of $\mathrm{G}$ with biomembranes to assess biological safety or toxicity of $\mathrm{G}$, as well as its promising function as a vector of new classes of antibiotics.

3. DNA or protein detection by $\mathrm{G}$ nanopores, an encouraging class of nanosensors that are less sensitive than biological pores to various factors such as the temperature and $\mathrm{pH}$.

In the next paragraph, we describe in more detail some of the studies of points 1 and 2 above, while for those in point 3 we refer the interested reader to the following works for the study of DNA detection (Sathe et al., 2011, 2014; Wells et al., 2012; Qiu et al., 2015; Barati Farimani et al., 2017a) and for the study of protein detection to Barati Farimani et al. (2017b).

\section{Adsorption of Biomolecules Onto Graphene}

\section{Pristine Graphene Substrates}

One of the first efforts involving all-atom MD simulations to study protein adsorption on Graphene is described in Zuo et al. (2011), where the villin headpiece (HP35) was employed. The simulations showed a rapid adsorption of HP35 on the substrate, causing relevant conformational changes in both secondary and tertiary protein structure. The $\pi / \pi$ stacking interactions between aromatic residues and $\mathrm{G}$ dominate the protein- $\mathrm{G}$ interaction, differently from what observed in simulations of HP35 with curved carbon nanostructures.

The properties of $G$ as a substrate for the adsorption of biomolecules have been investigated in subsequent years. Katoch et al. (2012) performed MD simulations, in combination with experimental techniques such as atomic force microscopy, Raman spectroscopy, and infrared spectroscopy to elucidate peptide-binding to $G$ and graphite. In this work, a dodecamer peptide forms a complex reticular structure upon adsorption, with a helical conformation different from $\alpha$-helix. Cheng et al. (2013) investigated via MD simulations the interaction between different peptides and G. In these simulations, a flat $G$ substrate becomes distorted after peptide adsorption. The authors conclude that multiple factors, including peptide size, number, distribution, and alignment, can affect the interaction with the $\mathrm{G}$ substrate. Zhou and collaborators ( $\mathrm{Gu}$ et al., 2015) performed MD simulations to show how blood proteins such as bovine fibrinogen (BFG) can rapidly adsorb onto the G surface. Markedly, these simulations describe, in addition to the aforementioned effect of strong $\pi / \pi$ stacking interactions, another key interaction due to basic residues. These residues play a relevant role during the process because of the strong dispersion interactions between their side-chains and the substrate. Globally, hydrophobic, electrostatic and $\pi / \pi$ stacking interactions drive the immobilization of the molecule on G.

Kim et al. (2015) examined the recognition of $\mathrm{G}$ by peptides with respect to the chemical composition of $G$, the number of over-imposed layers, and the underlying substrate support. The results of this computational work, together with experimental data based on Resonance Raman Spectroscopy, Quartz Crystal Microbalance and Water Contact Angle measurements, indicate that $\mathrm{G}$ quality is a significant factor in G-peptide interactions, while the interaction appears to show no significant dependency on the number of $\mathrm{G}$ layers or the underlying support substrate. Cheng et al. (2013) performed MD simulations between different peptides of silk fibroin and G (Cheng et al., 2015). This work illustrates that while $\mathrm{G}$ competes with the protein intramolecular interactions, reducing their $\beta$-sheet content, it also enhances the stability of segments with scarce ordered secondary structures and weak intramolecular interactions. Globally, G produces remarkable effects on the molecular conformation of these representative sequences.

Furthermore, Hughes and Walsh (2015) used enhancedsampling MD methods to investigate the links between the sequence and binding of adsorbed peptides onto G. First, the free energy of adsorption of all 20 naturally occurring amino acids was obtained using Well-Tempered Metadynamics (Barducci et al., 2008), thus providing a benchmark for interpreting peptide-graphene adsorption studies. In these calculations, strong binding is observed for amino acids which have flat and/or compact side chains. Then, replica exchange with solute tempering simulations (Terakawa et al., 2011) were performed to sample the conformational ensemble of two experimentally-characterized peptides, $\mathrm{P} 1$ and its alanine mutant P1A3, in solution and adsorbed on G. Results show that while P1A3 presents mostly disordered conformations, both in solution and when adsorbed, a helical P1 conformation is stabilized by adsorption on $\mathrm{G}$ via interaction of the stronglybinding residues.

In 2016, Yeo et al. (2016) investigated the adsorption mechanism of single and multiple bovine serum albumin (BSA) peptide segments on $G$, through the analysis of a broad set of parameters such as root-mean-square displacement, number of hydrogen bonds, helical content, interaction energies, and peptide center-of-mass displacement. The authors observed a destabilization of the helical structures in the single segment system, due to strong interactions between the peptide and the substrate. On the other hand, a better conservation in the total helical content is observed in the multi-segment system, implying a protective collective action of the peptides. More recently, No et al. (2017) reported nature-inspired two-dimensional peptide self-assembly on $G$ via optimization of peptide-peptide and peptide- $\mathrm{G}$ interactions. Atomistic simulations determined the optimal peptide sequence that leads to peptide self-assembly on G, suggesting that the optimal peptide sequence minimizes the peptide $-\mathrm{G}$ interaction energy and also the peptide-peptide interaction energy, resulting in stable complexes on $\mathrm{G}$.

\section{GO Substrates}

The adhesion of biomolecules to $\mathrm{GO}$ and $\mathrm{rGO}$ layers was investigated in Chong et al. (2015) to test the different advantages of GO due to the presence of the oxygen atoms. The interactions of serum proteins with GO nanosheets were explored with a large set of experimental techniques and with MD simulations, showing high adsorption capacities of GO and rGO. However, it is important to point out that while $\mathrm{GO}$ and rGO were used in the experiments, pristine $\mathrm{G}$ was chosen to simulate the relevant non-oxidized regions of the 
surface present on GO nanosheets. The action of GO was investigated more explicitly by representing the substrate using the Lerf-Klinowski model (Lerf et al., 1998), which describes the behavior of a standard oxidation process. Using this approach, two paradigmatic articles (Sun et al., 2014; Zeng et al., 2016) demonstrated that GO displays an enhanced adsorption of the attached protein. Firstly, in Sun et al. (2014), an atomistic description of the inhibitory action of GO on the activity of $\alpha$-chymotrypsin (ChT), has been provided. The results support the hypothesis that GO can be considered as a promising receptor for enzyme inhibition. Secondly, Zeng et al. (2016) show the details of the binding energy of GO to Vpr13-33, a fragment of the viral protein $\mathrm{R}(\mathrm{Vpr})$, using potential of mean force (PMF) calculations with the enhanced method umbrella sampling (Kästner, 2011).

Recently, Willems et al. (2017) investigated the dynamics of supported phospholipid membrane patches stabilized on $\mathrm{G}$ surfaces. These systems show potential in sensor device functionalization. The authors integrated experimental measures and CGMD simulations to characterize the molecular properties of supported lipid membranes (SLMs) on G and GO supports. The results described substantial differences in the topologies of the stabilized lipid structures, depending on the nature of the surface, providing novel details into the molecular effects of $G$ and $\mathrm{G}$ oxide surfaces on lipid membranes.

Overall, parallel to this considerable amount of data, in this emerging area of computational applications many fundamental issues remain unresolved, due to the lack of sufficient experimental results. In particular, the detailed distribution of the oxygen-containing groups on the substrate is difficult to determine, with a considerable loss in the description of the adsorption mechanism.

\section{Interactions of Graphene With Biomembranes}

The interaction of $\mathrm{G}$ with biomolecular complexes is crucial to understand its biological safety and potential toxicity. A seminal work (Tu et al., 2013) showed that GR and GO nanosheets induce the degradation of the inner and outer cell membranes of Escherichia Coli. Specifically, MD simulations showed that $\mathrm{G}$ is able to actively extract phospholipid molecules from a lipid bilayer, fixing them on its surface. Although these results introduce $\mathrm{G}$ as a convenient tool able to kill bacteria, there is an abundant literature where $\mathrm{G}$ also shows destructive capacities towards some biomolecules (Luan et al., 2015).

In this context, the results from CGMD simulations describe a quite different scenario. One of the first uses of CGMD for studying G-biomolecules interactions can be found in Titov et al. (2010). There, the Martini force field (Marrink et al., 2007) is used to study the interaction of $G$ nanosheets with phospholipid bilayers formed by 1-palmitoyl-2-oleoyl-snglycero-3-phosphocholine (POPC membrane). Results showed that $G$ sheets are hosted in the hydrophobic interior of the membrane, forming stable G-lipid structures (Figure 6).

In the following years, other works have investigated these systems with various CGMD algorithms, such as Guo et al. (2013), Li Y. et al. (2013) and Mao et al. (2014). In all these studies, however, lipid extraction or membrane damage is not observed, in contrast to the results of Tu et al. (2013). More recently, computational simulations were used to elucidate whether G causes cell membrane damage (Chen J. et al., 2016). All-atom MD simulations were used to study the interaction of both $\mathrm{G}$ and $\mathrm{GO}$ with respect to a dipalmitoylphosphatidylcholine (DPPC) bilayer, and they revealed that G quickly enters into the membrane by assuming a position parallel to the lipid tails. Conversely, GO did not enter the membrane spontaneously during the observed time-scale, but when docked onto the bilayer, it formed pores in the membrane.

A particularly important biomembrane system is the one involved in the formation of biological barriers such as for example the $\mathrm{BBB}$. Computational studies can be useful to investigate the effect of barrier exposure to G-based materials (Figure 6), but studying such complex architectures is still hampered by the lack of structural information (Alberini et al., 2017).

\section{CONCLUSIONS: FUTURE CHALLENGES AND PERSPECTIVES}

In the past few years, GRMs have been studied and used in a wide range of technological fields, including biomedical applications. The treatment of neurological disorders through non-invasive pharmacological approaches is still a major challenge. It is crucial for scientist to develop strategies for efficient cargo delivery of drugs or biomolecules or even genes to the brain, bypassing the $\mathrm{BBB}$ while preserving its structure and vital functions. One of the purposes of nanomedicine is indeed to create innovative ways for cell-targeting and drug-controlled release by avoiding surgery or other approaches that are very invasive for the patient. In this scenario, the choice of the appropriate ligand-receptor complex is a key design element when constructing nano-carriers, as well as the choice of the material, the size and eventual functionalization. While receptor-mediated transcytosis is a fundamental pathway for $\mathrm{BBB}$ crossing, the development of next-generation nano-carriers, like 2D-materials, and the investigation and optimization of alternative routes for delivery, such as intranasal administration, is of utmost importance for the scientific community.

Besides the "BBB challenge", other aspects of neuroscience could benefit of the latest developments in graphene research. Neuro-oncology may profit from the development of $G$ nanosheets and G NPs for tumor-targeted imaging, photothermal therapy, and anticancer drug delivery and gene therapy. New electrical, chemical and optical sensors may have great impact for neuro-intensive care and neuro-monitoring. Moreover, the combination of different forms and states of $\mathrm{G}$, diverse chemical functionalization and the possible association with other biomaterials to form G-based composites, may allow to devise an all-in one tool for both diagnosis and therapy, thus effectively building a powerful theranostic device.

Finally, tissue-engineering research is expected to develop novel brain-implant interfaces based on $\mathrm{G}$, to exploit the material 
electrical conductivity and enhance cell-cell communication and repair. Besides the experimental and clinical evidence, MD studies are emerging as an important aspect of material research, as they provide extremely precise indications and predictions on G/cell and G/protein interactions, guiding the researcher to design more powerful G-based devices.

Nevertheless, despite initial studies demonstrated the biocompatibility of $\mathrm{G}$, especially when conjugated with other materials in $2 \mathrm{D}$ and $3 \mathrm{D}$ scaffolds, only few systems were demonstrated to be successful in vivo. Further investigations are still required, in particular about the biological effects of long-term treatment with $G$ materials, before the promised technological applications can be fully exploited in and beyond neuroscience.

\section{REFERENCES}

Abbott, N. J. (2013). Blood-brain barrier structure and function and the challenges for CNS drug delivery. J. Inherit. Metab. Dis. 36, 437-449. doi: 10.1007/s10545013-9608-0

Abbott, N. J., Patabendige, A. A., Dolman, D. E., Yusof, S. R., and Begley, D. J. (2010). Structure and function of the blood-brain barrier. Neurobiol. Dis. 37, 13-25. doi: 10.1016/j.nbd.2009.07.030

Abbott, N. J., and Romero, I. A. (1996). Transporting therapeutics across the blood-brain barrier. Mol. Med. Today 2, 106-113. doi: 10.1016/13574310(96)88720-x

Abbott, N. J., Ronnback, L., and Hansson, E. (2006). Astrocyte-endothelial interactions at the blood-brain barrier. Nat. Rev. Neurosci. 7, 41-53. doi: $10.1038 / \mathrm{nrn} 1824$

Åberg, C. (2016). Quantitative analysis of nanoparticle transport through in vitro blood-brain barrier models. Tissue Barriers 4:e1143545. doi: 10.1080/21688370. 2016.1143545

Abraham, M. J., Murtola, T., Schultz, R., Pall, S., Smith, J. C., Hess, B., et al. (2015). High performance molecular simulations through multi-level parallelism from laptops to supercomputers. SoftwareX 1-2, 19-25. doi: 10.1016/j.softx.2015. 06.001

Akhavan, O., Ghaderi, E., and Akhavan, A. (2012). Size-dependent genotoxicity of graphene nanoplatelets in human stem cells. Biomaterials 33, 8017-8025. doi: 10.1016/j.biomaterials.2012.07.040

Akhavan, O., Ghaderi, E., Shirazian, S. A., and Rahighi, R. (2016). Rolled graphene oxide foams as three-dimensional scaffolds for growth of neural fibers using electrical stimulation of stem cells. Carbon 97, 71-77. doi: 10.1016/j.carbon. 2015.06.079

Alberini, G., Benfenati, F., and Maragliano, L. (2017). A refined model of claudin-15 tight junction paracellular architecture by molecular dynamics simulations. PLoS One 12:e0184190. doi: 10.1371/journal.pone.0184190

Allen, T. M., and Cullis, P. R. (2004). Drug delivery systems: entering the mainstream. Science 303, 1818-1822. doi: 10.1126/science.1095833

Ambrosi, A., and Pumera, M. (2016). Electrochemically exfoliated graphene and graphene oxide for energy storage and electrochemistry applications. Chemistry 22, 153-159. doi: 10.1002/chem.201503110

Andrieux, K., and Couvreur, P. (2009). Polyalkylcyanoacrylate nanoparticles for delivery of drugs across the blood-brain barrier. Wiley Interdiscip. Rev. Nanomed. Nanobiotechnol. 1, 463-474. doi: 10.1002/wnan.5

Apollo, N. V., Maturana, M. I., Tong, W., Nayagam, D. A. X., Shivdasani, M. N., Foroughi, J., et al. (2015). Soft, flexible freestanding neural stimulation and recording electrodes fabricated from reduced graphene oxide. Adv. Funct. Mater. 25, 3551-3559. doi: 10.1002/adfm.201500110

Baldrighi, M., Trusel, M., Tonini, R., and Giordani, S. (2016). Carbon nanomaterials interfacing with neurons: an in vivo perspective. Front. Neurosci. 10:250. doi: 10.3389/fnins.2016.00250

Barati Farimani, A., Dibaeinia, P., and Aluru, N. R. (2017a). DNA origamigraphene hybrid nanopore for DNA detection. ACS Appl. Mater. Interfaces 9, 92-100. doi: 10.1021/acsami.6b11001

\section{AUTHOR CONTRIBUTIONS}

$\mathrm{MB}, \mathrm{FC}$ and $\mathrm{FB}$ conceived, structured and wrote the review. All authors participated in writing the review and specifically MC contributed to the Introduction section. EC, MLD and JFM-V contributed to the section of neuronal recording and stimulation. GA and LM contributed to the molecular dynamics and modeling section.

\section{ACKNOWLEDGMENTS}

The authors acknowledge financial support from EU H2020 research and innovation programme under grant agreement no. 696656 (Graphene Flaghship-Core1).

Barati Farimani, A., Heiranian, M., Min, K., and Aluru, N. R. (2017b). Antibody subclass detection using graphene nanopores. J. Phys. Chem. Lett. 8, 1670-1676. doi: 10.1021/acs.jpclett.7b00385

Barducci, A., Bussi, G., and Parrinello, M. (2008). Well-tempered metadynamics: a smoothly converging and tunable free-energy method. Phys. Rev. Lett. 100:020603. doi: 10.1103/physrevlett.100.020603

Begley, D. J. (2004). ABC transporters and the blood-brain barrier. Curr. Pharm. Des. 10, 1295-1312. doi: 10.2174/1381612043384844

Begley, D. J., and Brightman, M. W. (2003). Structural and functional aspects of the blood-brain barrier. Prog. Drug Res. 61, 39-78. doi: 10.1007/978-3-03488049-7 2

Bianco, A. (2013). Graphene: safe or toxic? The two faces of the medal. Angew. Chem. Int. Ed Engl. 52, 4986-4997. doi: 10.1002/anie.201209099

Bitounis, D., Ali-Boucetta, H., Hong, B. H., Min, D. H., and Kostarelos, K. (2013). Prospects and challenges of graphene in biomedical applications. Adv. Mater. 25, 2258-2268. doi: 10.1002/adma.201203700

Blaschke, B. M., Lottner, M., Drieschner, S., Bonaccini Calia, A., Stoiber, K., Rousseau, L., et al. (2016). Flexible graphene transistor for recording cell action potentials. 2d Mater. 3:025007. doi: 10.1088/2053-1583/3/2/025007

Bonaccorso, F., Bartolotta, A., Coleman, J. N., and Backes, C. (2016). 2D-crystalbased functional inks. Adv. Mater. 28, 6136-6166. doi: 10.1002/adma 201506410

Bonaccorso, F., Colombo, L., Yu, G., Stoller, M., Tozzini, V., Ferrari, A. C., et al. (2015). 2D materials. Graphene, related two-dimensional crystals, and hybrid systems for energy conversion and storage. Science 347:1246501. doi: $10.1126 /$ science. 1246501

Bramini, M., Alberini, G., Benfenati, F., Maragliano, L., and Cesca, F. (2018). "Interactions between 2D graphene-based materials and the nervous tissue," in 2D Materials: Characterization, Production and Application, ed. C. E. Brownson (Boca Raton, FL: CRC Press, Taylor and Francis Group), $62-85$.

Bramini, M., Sacchetti, S., Armirotti, A., Rocchi, A., Vázquez, E., León Castellanos, V., et al. (2016). Graphene oxide nanosheets disrupt lipid composition, $\mathrm{Ca}^{2+}$ homeostasis, and synaptic transmission in primary cortical neurons. ACS Nano 10, 7154-7171. doi: 10.1021/acsnano.6b03438

Bramini, M., Ye, D., Hallerbach, A., Nic Raghnaill, M., Salvati, A., Aberg, C., et al (2014). Imaging approach to mechanistic study of nanoparticle interactions with the blood-brain barrier. ACS Nano 8, 4304-4312. doi: 10.1021/nn5018523

Brooks, B. R., Brooks, C. L. III., Mackerell, A. D. Jr., Nilsson, L., Petrella, R. J., Roux, B., et al. (2009). CHARMM: the biomolecular simulation program. J. Comput. Chem. 30, 1545-1614. doi: 10.1002/jcc.21287

Casaluci, S., Gemmi, M., Pellegrini, V., Di Carlo, A., and Bonaccorso, F. (2016). Graphene-based large area dye-sensitized solar cell modules. Nanoscale 8, 5368-5378. doi: 10.1039/c5nr07971c

Cavallucci, T., Kakhiani, K., Farchioni, R., and Tozzini, V. (2016). Morphing graphene-based systems for applications: perspectives from simulation. arXiv:1609.07871

Chang, E. F. (2015). Towards large-scale, human-based, mesoscopic neurotechnologies. Neuron 86, 68-78. doi: 10.1016/j.neuron.2015.03.037 
Chen, R., Canales, A., and Polina, A. (2017). Neural recording and modulation technologies. Nat. Rev. Mater. 2:16093. doi: 10.1038/natrevmats.2016.93

Chen, G. Y., Chen, C. L., Tuan, H. Y., Yuan, P. X., Li, K. C., Yang, H. J., et al. (2014). Graphene oxide triggers toll-like receptors/autophagy responses in vitro and inhibits tumor growth in vivo. Adv. Healthc. Mater. 3, 1486-1495. doi: 10.1002/adhm.201300591

Chen, B., Liu, M., Zhang, L., Huang, J., Yao, J., and Zhang, Z. (2011). Polyethyleniimine-functionalized graphene oxide as an efficient gene delivery vector. J. Mater. Chem. 11, 7736-7741. doi: 10.1039/c1jm10341e

Chen, Y., Star, A., and Vidal, S. (2013). Sweet carbon nanostructures: carbohydrate conjugates with carbon nanotubes and graphene and their applications. Chem. Soc. Rev. 42, 4532-4542. doi: 10.1039/c2cs35396b

Chen, M., Yin, J., Liang, Y., Yuan, S., Wang, F., Song, M., et al. (2016). Oxidative stress and immunotoxicity induced by graphene oxide in zebrafish. Aquat. Toxicol. 174, 54-60. doi: 10.1016/j.aquatox.2016.02.015

Chen, J., Zhou, G., Chen, L., Wang, Y., Wang, X., and Zeng, S. (2016). Interaction of graphene and its oxide with lipid membrane: a molecular dynamics simulation study. J. Phys. Chem. C 120, 6225-6231. doi: 10.1021/acs.jpcc. 5b10635

Cheng, Y., Koh, L. D., Li, D., Ji, B., Zhang, Y., Yeo, J., et al. (2015). Peptidegraphene interactions enhance the mechanical properties of silk fibroin. ACS Appl. Mater. Interfaces 7, 21787-21796. doi: 10.1021/acsami.5b05615

Cheng, C., Li, S., Thomas, A., Kotov, A. N., and Haag, R. (2016). Functional graphene nanomaterials based architectures: biointeractions, fabrications, and emerging biological applications. Chem. Rev. 117, 1826-1914. doi: 10.1021/acs. chemrev.6b00520

Cheng, Y., Zhang, Z., and Teo, Z. (2013). Deformation of graphene induced by adsorption of peptides: a molecular dynamics study. Int. J. Appl. Mech. 5:1350007. doi: 10.1142/s1758825113500075

Choi, M., Ku, T., Chong, K., Yoon, J., and Choi, C. (2011). Minimally invasive molecular delivery into the brain using optical modulation of vascular permeability. Proc. Natl. Acad. Sci. U S A 108, 9256-9261. doi: 10.1073/pnas. 1018790108

Chong, Y., Ge, C., Yang, Z., Garate, J. A., Gu, Z., Weber, J. K., et al. (2015). Reduced cytotoxicity of graphene nanosheets mediated by blood-protein coating. ACS Nano 9, 5713-5724. doi: 10.1021/nn5066606

Chowdhury, I., Duch, M. C., Mansukhani, N. D., Hersam, M. C., and Bouchard, D. (2013). Colloidal properties and stability of graphene oxide nanomaterials in the aquatic environment. Environ. Sci. Technol. 47, 6288-6296. doi: 10.1021/es400483k

Clark, A. J., and Davis, M. E. (2015). Increased brain uptake of targeted nanoparticles by adding an acid-cleavable linkage between transferrin and the nanoparticle core. Proc. Natl. Acad. Sci. U S A 112, 12486-12491. doi: 10.1073/pnas.1517048112

Cong, H. P., Chen, J. F., and Yu, S. H. (2014). Graphene-based macroscopic assemblies and architectures: an emerging material system. Chem. Soc. Rev. 43, 7295-7325. doi: 10.1039/c4cs00181h

Crowder, S. W., Prasai, D., Rath, R., Balikov, D. A., Bae, H., Bolotin, K. I., et al. (2013). Three-dimensional graphene foams promote osteogenic differentiation of human mesenchymal stem cells. Nanoscale 5, 4171-4176. doi: $10.1039 / \mathrm{c} 3 \mathrm{nr} 00803 \mathrm{~g}$

Defterali, Ç., Verdejo, R., Majeed, S., Boschetti-De-Fierro, A., MendezGomez, H. R., Diaz-Guerra, E., et al. (2016a). In vitro evaluation of biocompatibility of uncoated thermally reduced graphene and carbon nanotube-loaded PVDF membranes with adult neural stem cell-derived neurons and glia. Front. Bioeng. Biotechnol. 4:94. doi: 10.3389/fbioe.2016.00094

Defterali, Ç., Verdejo, R., Peponi, L., Martín, E. D., Martínez-Murillo, R., LópezManchado, M. A., et al. (2016b). Thermally reduced graphene is a permissive material for neurons and astrocytes and de novo neurogenesis in the adult olfactory bulb in vivo. Biomaterials 82, 84-93. doi: 10.1016/j.biomaterials.2015. 12.010

Dell'Orco, D., Lundqvist, M., Oslakovic, C., Cedervall, T., and Linse, S. (2010). Modeling the time evolution of the nanoparticle-protein corona in a body fluid. PLoS One 5:e10949. doi: 10.1371/journal.pone.0010949

Ding, X., Liu, H., and Fan, Y. (2015). Graphene-based materials in regenerative medicine. Adv. Healthc. Mater. 4, 1451-1468. doi: 10.1002/adhm.201500203

Dohgu, S., Takata, F., Yamauchi, A., Nakagawa, S., Egawa, T., Naito, M., et al. (2005). Brain pericytes contribute to the induction and up-regulation of blood-brain barrier functions through transforming growth factor- $\beta$ production. Brain Res. 1038, 208-215. doi: 10.1016/j.brainres.2005.01.027

Donaldson, K., Aitken, R., Tran, L., Stone, V., Duffin, R., Forrest, G., et al. (2006). Carbon nanotubes: a review of their properties in relation to pulmonary toxicology and workplace safety. Toxicol. Sci. 92, 5-22. doi: 10.1093/toxsci/kfj130

Dong, H., Jin, M., Liu, Z., Xiong, H., Qiu, X., Zhang, W., et al. (2016). In vitro and in vivo brain-targeting chemo-photothermal therapy using graphene oxide conjugated with transferrin for Gliomas. Lasers Med. Sci. 31, 1123-1131. doi: 10.1007/s10103-016-1955-2

Du, D., Zou, Z., Shin, Y., Wang, J., Wu, H., Engelhard, M. H., et al. (2010). Sensitive immunosensor for cancer biomarker based on dual signal amplification strategy of graphene sheets and multienzyme functionalized carbon nanospheres. Anal. Chem. 82, 2989-2995. doi: 10.1021/ac100036p

Dufficy, M. K., Geiger, M. T., Bonino, C. A., and Khan, S. A. (2015). Electrospun ultrafine fiber composites containing fumed silica: from solution rheology to materials with tunable wetting. Langmuir 31, 12455-12463. doi: 10.1021/acs. langmuir.5b03545

Eda, G., Fanchini, G., and Chhowalla, M. (2008). Large-area ultrathin films of reduced graphene oxide as a transparent and flexible electronic material. Nat. Nanotechnol. 3, 270-274. doi: 10.1038/nnano.2008.83

Ek, C. J., Dziegielewska, K. M., Stolp, H., and Saunders, N. R. (2006). Functional effectiveness of the blood-brain barrier to small water-soluble molecules in developing and adult opossum (Monodelphis domestica). J. Comp. Neurol. 496, 13-26. doi: 10.1002/cne.20885

El-Kady, M. F., and Kaner, R. B. (2013). Scalable fabrication of high-power graphene micro-supercapacitors for flexible and on-chip energy storage. Nat. Commun. 4:1475. doi: 10.1038/ncomms 2446

Fabbro, A., Scaini, D., León, V., Vázquez, E., Cellot, G., Privitera, G., et al. (2016). Graphene-based interfaces do not alter target nerve cells. ACS Nano 10, 615-623. doi: 10.1021/acsnano.5b05647

Fahmi, T., Branch, D., Nima, Z. A., Jang, D. S., Savenka, A. V., Biris, A. S., et al. (2017). Mechanism of graphene-induced cytotoxicity: role of endonucleases. J. Appl. Toxicol. 37, 1325-1332. doi: 10.1002/jat.3462

Feng, L., Wu, L., and Qu, X. (2013). New horizons for diagnostics and therapeutic applications of graphene and graphene oxide. Adv. Mater. 25, 168-186. doi: 10.1002/adma.201203229

Feng, L., Zhang, S., and Liu, Z. (2011). Graphene based gene transfection. Nanoscale 3, 1252-1257. doi: 10.1039/c0nr00680g

Gao, X., Qian, J., Zheng, S., Changyi, Y., Zhang, J., Ju, S., et al. (2014). Overcoming the blood-brain barrier for delivering drugs into the brain by using adenosine receptor nanoagonist. ACS Nano 8, 3678-3689. doi: 10.1021/nn5003375

Geim, A. K. (2009). Graphene: status and prospects. Science 324, 1530-1534. doi: $10.1126 /$ science. 1158877

Gelperina, S., Maksimenko, O., Khalansky, A., Vanchugova, L., Shipulo, E., Abbasova, K., et al. (2010). Drug delivery to the brain using surfactantcoated poly(lactide-co-glycolide) nanoparticles: influence of the formulation parameters. Eur. J. Pharm. Biopharm. 74, 157-163. doi: 10.1016/j.ejpb.2009. 09.003

Georgakilas, V., Tiwari, J. N., Kemp, K. C., Perman, J. A., Bourlinos, A. B., Kim, K. S., et al. (2016). Noncovalent functionalization of graphene and graphene oxide for energy materials, biosensing, catalytic, and biomedical applications. Chem. Rev. 116, 5464-5519. doi: 10.1021/acs.chemrev.5b00620

Goenka, S., Sant, V., and Sant, S. (2014). Graphene-based nanomaterials for drug delivery and tissue engineering. J. Control. Release 173, 75-88. doi: 10.1016/j. jconrel.2013.10.017

Gollavelli, G., and Ling, Y. C. (2012). Multi-functional graphene as an in vitro and in vivo imaging probe. Biomaterials 33, 2532-2545. doi: 10.1016/j.biomaterials. 2011.12.010

Gu, Z., Yang, Z., Wang, L., Zhou, H., Jimenez-Cruz, C. A., and Zhou, R. (2015). The role of basic residues in the adsorption of blood proteins onto the graphene surface. ACS Nano 5:10873. doi: 10.1038/srep10873

Guo, R., Mao, J., and Yan, L. T. (2013). Computer simulation of cell entry of graphene nanosheet. Biomaterials 34, 4296-4301. doi: 10.1016/j.biomaterials. 2013.02.047

Guo, W., Qiu, J., Liu, J., and Liu, H. (2017). Graphene microfiber as a scaffold for regulation of neural stem cells differentiation. Sci. Rep. 7:5678. doi: 10.1038/s41598-017-06051-z 
Guo, W., Wang, S., Yu, X., Qiu, J., Li, J., Tang, W., et al. (2016). Construction of a 3D rGO-collagen hybrid scaffold for enhancement of the neural differentiation of mesenchymal stem cells. Nanoscale 8, 1897-1904. doi: 10.1039/c5nr06602f

Hassoun, J., Bonaccorso, F., Agostini, M., Angelucci, M., Betti, M. G., Cingolani, R., et al. (2014). An advanced lithium-ion battery based on a graphene anode and a lithium iron phosphate cathode. Nano Lett. 14, 4901-4906. doi: 10.1021/nl502429m

Hatsopoulos, N. G., and Donoghue, J. P. (2009). The science of neural interface systems. Annu. Rev. Neurosci. 32, 249-266. doi: 10.1146/annurev.neuro. 051508.135241

Hawkins, R. A., O'Kane, R. L., Simpson, I. A., and Viña, J. R. (2006). Structure of the blood-brain barrier and its role in the transport of amino acids. J. Nutr. 136, 218S-226S. doi: 10.1093/jn/136.1.218S

Herda, L. M., Polo, E., Kelly, P. M., Rocks, L., Hudecz, D., and Dawson, K. A. (2014). Designing the future of nanomedicine: current barriers to targeted brain therapeutics. Eur. J. Nanomed. 6, 127-139. doi: 10.1515/ejnm-2014-0022

Hess, L. H., Jansen, M., Maybeck, V., Hauf, M. V., Seifert, M., Stutzmann, M., et al. (2011). Graphene transistor arrays for recording action potentials from electrogenic cells. Adv. Mater. Weinheim. 23, 5045-5049, 4968. doi: 10.1002/adma.201102990

Hönigsmann, H. (2013). History of phototherapy in dermatology. Photochem. Photobiol. Sci. 12, 16-21. doi: 10.1039/c2pp25120e

Hossain, R. F., Deaguero, I. G., Boland, T., and Kaul, A. B. (2017). Biocompatible, large-format, inkjet printed heterostructure MoS2-graphene photodetectors on conformable substrates. npj 2D Mater. Appl. 28, 1-10. doi: 10.1038/s41699-0170034-2

Hsieh, T. Y., Huang, W. C., Kang, Y. D., Chu, C. Y., Liao, W. L., Chen, Y. Y., et al. (2016). Neurotensin-conjugated reduced graphene oxide with multi-stage near-infrared-triggered synergic targeted neuron gene transfection in vitro and in vivo for neurodegenerative disease therapy. Adv. Healthc. Mater. 5, 3016-3026. doi: 10.1002/adhm.201600647

Hu, N., Zhang, L., Yang, C., Zhao, J., Yang, Z., Wei, H., et al. (2016). Threedimensional skeleton networks of graphene wrapped polyaniline nanofibers: an excellent structure for high-performance flexible solid-state supercapacitors. Sci. Rep. 6:19777. doi: 10.1038/srep19777

Hughes, Z. E., and Walsh, T. R. (2015). What makes a good graphenebinding peptide? Adsorption of amino acids and peptides at aqueous graphene interfaces. J. Mater. Chem. B 3, 3211-3221. doi: 10.1039/c5tb0 $0004 \mathrm{a}$

Hummer, G., Rasaiah, J. C., and Noworyta, J. P. (2001). Water conduction through the hydrophobic channel of a carbon nanotube. Nature 414, 188-190. doi: $10.1038 / 35102535$

Jiang, Z., Song, Q., Tang, M., Yang, L., Cheng, Y., Zhang, M., et al. (2016). Enhanced migration of neural stem cells by microglia grown on a threedimensional graphene scaffold. ACS Appl. Mater. Interfaces 8, 25069-25077. doi: 10.1021/acsami.6b06780

John, A. A., Subramanian, A. P., Vellayappan, M. V., Balaji, A., Mohandas, H., and Jaganathan, S. K. (2015). Carbon nanotubes and graphene as emerging candidates in neuroregeneration and neurodrug delivery. Int. J. Nanomedicine 10, 4267-4277. doi: 10.2147/IJN.S83777

Kanakia, S., Toussaint, J. D., Mullick Chowdhury, S., Tembulkar, T., Lee, S., Jiang, Y. P., et al. (2014). Dose ranging, expanded acute toxicity and safety pharmacology studies for intravenously administered functionalized graphene nanoparticle formulations. Biomaterials 35, 7022-7031. doi: 10.1016/j. biomaterials.2014.04.066

Kang, P., Wang, M. C., and Nam, S. (2016). Bioelectronics with two-dimensional materials. Microelectron. Eng. 161, 18-35. doi: 10.1016/j.mee.2016.04.003

Kang, X., Wang, J., Wu, H., Liu, J., Aksay, I. A., and Lin, Y. (2010). A graphenebased electrochemical sensor for sensitive detection of paracetamol. Talanta 81, 754-759. doi: 10.1016/j.talanta.2010.01.009

Kästner, J. (2011). Umbrella sampling. Adv. Rev. 1, 932-942. doi: 10.1002/ wcms.66

Katoch, J., Kim, S. N., Kuang, Z., Farmer, B. L., Naik, R. R., Tatulian, S. A., et al. (2012). Structure of a peptide adsorbed on graphene and graphite. Nano Lett. 12, 2342-2346. doi: 10.1021/nl300286k

Kim, D. H., Abidian, M., and Martin, D. C. (2004). Conducting polymers grown in hydrogel scaffolds coated on neural prosthetic devices. J. Biomed. Mater. Res. A 71, 577-585. doi: 10.1002/jbm.a.30124
Kim, S. S., Kuang, Z., Ngo, Y. H., Farmer, B. L., and Naik, R. R. (2015). Biotic-abiotic interactions: factors that influence peptide-graphene interactions. ACS Appl. Mater. Interfaces 7, 20447-20453. doi: 10.1021/acsami. $5 \mathrm{~b} 06434$

Kim, D. H., and Martin, D. C. (2006). Sustained release of dexamethasone from hydrophilic matrices using PLGA nanoparticles for neural drug delivery. Biomaterials 27, 3031-3037. doi: 10.1016/j.biomaterials.2005. 12.021

Kim, H., Namgung, R., Singha, K., Oh, I. K., and Kim, W. J. (2011). Graphene oxide-polyethylenimine nanoconstruct as a gene delivery vector and bioimaging tool. Bioconjug. Chem. 22, 2558-2567. doi: 10.1021/bc200397j

Kostarelos, K., Vincent, M., Hebert, C., and Garrido, J. A. (2017). Graphene in the design and engineering of next-generation neural interfaces. Adv. Mater. 29:1700909. doi: 10.1002/adma.201700909

Kreuter, J. (2014). Drug delivery to the central nervous system by polymeric nanoparticles: what do we know? Adv. Drug Deliv. Rev. 71, 2-14. doi: 10.1016/j. addr.2013.08.008

Kreuter, J., Ramge, P., Petrov, V., Hamm, S., Gelperina, S. E., Engelhardt, B., et al. (2003). Direct evidence that polysorbate-80-coated poly(butylcyanoacrylate) nanoparticles deliver drugs to the CNS via specific mechanisms requiring prior binding of drug to the nanoparticles. Pharm. Res. 20, 409-416. doi: 10.1023/A:1022604120952

Kreuter, J., Shamenkov, D., Petrov, V., Ramge, P., Cychutek, K., KochBrandt, C., et al. (2002). Apolipoprotein-mediated transport of nanoparticlebound drugs across the blood-brain barrier. J. Drug Target. 10, 317-325. doi: 10.1080/10611860290031877

Kuo, Y. C., and Chung, C. Y. (2012). Transcytosis of CRM197-grafted polybutylcyanoacrylate nanoparticles for delivering zidovudine across human brain-microvascular endothelial cells. Colloids Surf. B Biointerfaces 91, 242-249. doi: 10.1016/j.colsurfb.2011.11.007

Kuo, Y. C., and Liu, Y. C. (2014). Cardiolipin-incorporated liposomes with surface CRM197 for enhancing neuronal survival against neurotoxicity. Int. J. Pharm. 473, 334-344. doi: 10.1016/j.ijpharm.2014.07.003

Kurapati, R., Backes, C., Ménard-Moyon, C., Coleman, J. N., and Bianco, A. (2016). White graphene undergoes peroxidase degradation. Angew. Chem. Int. Ed Engl. 55, 5506-5511. doi: 10.1002/anie.201601238

Kuzum, D., Takano, H., Shim, E., Reed, J. C., Juul, H., Richardson, A. G., et al. (2014). Transparent and flexible low noise graphene electrodes for simultaneous electrophysiology and neuroimaging. Nat. Commun. 5:5259. doi: 10.1038/ncomms6259

Lerf, A., He, H., Forster, M., and Klinowski, J. (1998). Structure of graphite oxide revisited. J. Phys. Chem. B 102, 4477-4482. doi: 10.1021/jp97 31821

Li, N., Xiao, T., Zhang, Z., He, R., Wen, D., Cao, Y., et al. (2015). A 3D graphene oxide microchip and a Au-enwrapped silica nanocomposite-based supersandwich cytosensor toward capture and analysis of circulating tumor cells. Nanoscale 7, 16354-16360. doi: 10.1039/c5nr04798f

Li, Y., Yuan, H., von dem Bussche, A., Creighton, M., Hurt, R. H., Kane, A. B., et al. (2013). Graphene microsheets enter cells through spontaneous membrane penetration at edge asperities and corner sites. Proc. Natl. Acad. Sci. U S A 110 , 12295-12300. doi: 10.1073/pnas.1222276110

Li, N., Zhang, Q., Gao, S., Song, Q., Huang, R., Wang, L., et al. (2013). Threedimensional graphene foam as a biocompatible and conductive scaffold for neural stem cells. Sci. Rep. 3:1604. doi: 10.1038/srep01604

Li, N., Zhang, X., Song, Q., Su, R., Zhang, Q., Kong, T., et al. (2011). The promotion of neurite sprouting and outgrowth of mouse hippocampal cells in culture by graphene substrates. Biomaterials 32, 9374-9382. doi: 10.1016/j.biomaterials. 2011.08.065

Liao, K. H., Lin, Y. S., Macosko, C. W., and Haynes, C. L. (2011). Cytotoxicity of graphene oxide and graphene in human erythrocytes and skin fibroblasts. ACS Appl. Mater. Interfaces 3, 2607-2615. doi: 10.1021/am200428v

Lim, M. H., Jeung, I. C., Jeong, J., Yoon, S. J., Lee, S. H., Park, J., et al. (2016). Graphene oxide induces apoptotic cell death in endothelial cells by activating autophagy via calcium-dependent phosphorylation of c-Jun N-terminal kinases. Acta Biomater. 46, 191-203. doi: 10.1016/j.actbio.2016. 09.018

Liu, T. C., Chuang, M. C., Chu, C. Y., Huang, W. C., Lai, H. Y., Wang, C. T., et al. (2016). Implantable graphene-based neural electrode interfaces for 
electrophysiology and neurochemistry in in vivo hyperacute stroke model. ACS Appl. Mater. Interfaces 8, 187-196. doi: 10.1021/acsami.5b08327

Liu, J., Cui, L., and Losic, D. (2013). Graphene and graphene oxide as new nanocarriers for drug delivery applications. Acta Biomater. 9, 9243-9257. doi: 10.1016/j.actbio.2013.08.016

Liu, L., Guo, K., Lu, J., Venkatraman, S. S., Luo, D., Ng, K. C., et al. (2008a). Biologically active core/shell nanoparticles self-assembled from cholesterolterminated PEG-TAT for drug delivery across the blood-brain barrier. Biomaterials 29, 1509-1517. doi: 10.1016/j.biomaterials.2007.11.014

Liu, L., Venkatraman, S. S., Yang, Y. Y., Guo, K., Lu, J., He, B., et al. (2008b). Polymeric micelles anchored with TAT for delivery of antibiotics across the blood-brain barrier. Biopolymers 90, 617-623. doi: 10.1002/bip.20998

Liu, X., Ma, D., Tang, H., Tan, L., Xie, Q., Zhang, Y., et al. (2014). Polyamidoamine dendrimer and oleic acid-functionalized graphene as biocompatible and efficient gene delivery vectors. ACS Appl. Mater. Interfaces 6, 8173-8183. doi: $10.1021 / \mathrm{am} 500812 \mathrm{~h}$

Liu, Z., Robinson, J. T., Sun, X., and Dai, H. (2008). PEGylated nanographene oxide for delivery of water-insoluble cancer drugs. J. Am. Chem. Soc. 130, 10876-10877. doi: 10.1021/ja803688x

Liu, Z., Robinson, J. T., Tabakman, S. M., Yang, K., and Dai, H. (2011). Carbon materials for drug delivery and cancer therapy. Mater. Today 14, 316-323. doi: 10.1016/S1369-7021(11)70161-4

Liu, G., Shen, H., Mao, J., Zhang, L., Jiang, Z., Sun, T., et al. (2013). Transferrin modified graphene oxide for glioma-targeted drug delivery: in vitro and in vivo evaluations. ACS Appl. Mater. Interfaces 5, 6909-6914. doi: 10.1021/am4 $02128 \mathrm{~s}$

López-Dolado, E., González-Mayorga, A., Gutiérrez, M. C., and Serrano, M. C. (2016). Immunomodulatory and angiogenic responses induced by graphene oxide scaffolds in chronic spinal hemisected rats. Biomaterials 99, 72-81. doi: 10.1016/j.biomaterials.2016.05.012

López-Dolado, E., González-Mayorga, A., Portolés, M. T., Feito, M. J., Ferrer, M. L., Del Monte, F., et al. (2015). Subacute tissue response to 3d graphene oxide scaffolds implanted in the injured rat spinal cord. Adv. Healthc. Mater. 4, 1861-1868. doi: 10.1002/adhm.201500333

Loureiro, J. A., Gomes, B., Coelho, M. A., do Carmo Pereira, M., and Rocha, S. (2014). Targeting nanoparticles across the blood-brain barrier with monoclonal antibodies. Nanomedicine (Lond) 9, 709-722. doi: 10.2217/nnm.14.27

Lu, J., Cheng, C., He, Y. S., Lyu, C., Wang, Y., Yu, J., et al. (2016). Multilayered graphene hydrogel membranes for guided bone regeneration. Adv. Mater. 28, 4025-4031. doi: 10.1002/adma.201505375

Luan, B., Huynh, T., Zhao, L., and Zhou, R. (2015). Potential toxicity of graphene to cell functions via disrupting protein-protein interactions. ACS Nano 9, 663-669. doi: 10.1021/nn506011j

Lv, M., Zhang, Y., Liang, L., Wei, M., Hu, W., Li, X., et al. (2012). Effect of graphene oxide on undifferentiated and retinoic acid-differentiated SH-SY5Y cells line. Nanoscale 4, 3861-3866. doi: 10.1039/c2nr30407d

Ma, Q., Yang, L., Jiang, Z., Song, Q., Xiao, M., Zhang, D., et al. (2016). Threedimensional stiff graphene scaffold on neural stem cells behavior. ACS Appl. Mater. Interfaces 8, 34227-34233. doi: 10.1021/acsami.6b12305

Mahmoudifard, M., Soleimani, M., Hatamie, S., Zamanlui, S., Ranjbarvan, P., Vossoughi, M., et al. (2016). The different fate of satellite cells on conductive composite electrospun nanofibers with graphene and graphene oxide nanosheets. Biomed. Mater. 11:025006. doi: 10.1088/1748-6041/11/2/ 025006

Mao, J., Guo, R., and Yan, L. T. (2014). Simulation and analysis of cellular internalization pathways and membrane perturbation for graphene nanosheets. Biomaterials 35, 6069-6077. doi: 10.1016/j.biomaterials.2014. 03.087

Markovic, Z. M., Harhaji-Trajkovic, L. M., Todorovic-Markovic, B. M., Kepic, D. P., Arsikin, K. M., Jovanovic, S. P., et al. (2011). In vitro comparison of the photothermal anticancer activity of graphene nanoparticles and carbon nanotubes. Biomaterials 32, 1121-1129. doi: 10.1016/j.biomaterials.2010. 10.030

Marrink, S. J., Risselada, H. J., Yefimov, S., Tieleman, D. P., and de Vries, A. H. (2007). The MARTINI force field: coarse grained model for biomolecular simulations. J. Phys. Chem. B 111, 7812-7824. doi: 10.1021/jp071097f

Martín, C., Merino, S., Gonzalez-Dominguez, J. M., Rauti, R., Ballerini, L., Prato, M., et al. (2017). Graphene improves the biocompatibility of polyacrylamide hydrogels: $3 \mathrm{~d}$ polymeric scaffolds for neuronal growth. Sci. Rep. 7:10942. doi: 10.1038/s41598-017-11359-x

Mattei, T. A., and Rehman, A. A. (2014). Technological developments and future perspectives on graphene-based metamaterials: a primer for neurosurgeons. Neurosurgery 74, 499-516; discussion 516. doi: 10.1227/neu.0000000000 000302

McCallion, C., Burthem, J., Rees-Unwin, K., Golovanov, A., and Pluen, A. (2016). Graphene in therapeutics delivery: problems, solutions and future opportunities. Eur. J. Pharm. Biopharm. 104, 235-250. doi: 10.1016/j.ejpb.2016. 04.015

Medina-Sánchez, M., Miserere, S., and Merkoci, A. (2012). Nanomaterials and labon-a-chip technologies. Lab Chip 12, 1932-1943. doi: 10.1039/c2lc40063d

Menaa, F., Abdelghani, A., and Menaa, B. (2015). Graphene nanomaterials as biocompatible and conductive scaffolds for stem cells: impact for tissue engineering and regenerative medicine. J. Tissue Eng. Regen. Med. 9, 1321-1338. doi: 10.1002/term.1910

Mendonça, M. C., Soares, E. S., De Jesus, M. B., Ceragioli, H. J., Batista, Â. G., Nyúl-Tóth, Á., et al. (2016a). PEGylation of reduced graphene oxide induces toxicity in cells of the blood-brain barrier: an in vitro and in vivo study. Mol. Pharm. 13, 3913-3924. doi: 10.1021/acs.molpharmaceut.6b00696

Mendonça, M. C., Soares, E. S., De Jesus, M. B., Ceragioli, H. J., Irazusta, S. P., Batista, Â. G., et al. (2016b). Reduced graphene oxide: nanotoxicological profile in rats. J. Nanobiotechnology 14:53. doi: 10.1186/s12951-016-0206-9

Meric, I., Han, M. Y., Young, A. F., Ozyilmaz, B., Kim, P., and Shepard, K. L. (2008). Current saturation in zero-bandgap, top-gated graphene field-effect transistors. Nat. Nanotechnol. 3, 654-659. doi: 10.1038/nnano.2008.268

Michaelis, K., Hoffmann, M. M., Dreis, S., Herbert, E., Alyautdin, R. N., Michaelis, M., et al. (2006). Covalent linkage of apolipoprotein e to albumin nanoparticles strongly enhances drug transport into the brain. J. Pharmacol. Exp. Ther. 317, 1246-1253. doi: 10.1124/jpet.105.097139

Mittal, S., Kumar, V., Dhiman, N., Chauhan, L. K., Pasricha, R., and Pandey, A. K. (2016). Physico-chemical properties based differential toxicity of graphene oxide/reduced graphene oxide in human lung cells mediated through oxidative stress. Sci. Rep. 6:39548. doi: 10.1038/srep39548

Nieto, A., Dua, R., Zhang, C., Boesl, B., Ramaswamy, S., and Agarwal, A. (2015). Three dimensional graphene foam/polymer hybrid as a high strength biocompatible scaffold. Adv. Funct. Mater. 25, 3916-3924. doi: 10.1002/adfm. 201500876

No, Y. H., Kim, N. H., Gnapareddy, B., Choi, B., Kim, Y. T., Dugasani, S. R., et al. (2017). Nature-inspired construction of two-dimensionally self-assembled peptide on pristine graphene. J. Phys. Chem. Lett. 8, 3734-3739. doi: 10.1021/acs.jpclett.7b00996

Novoselov, K. S., Fal'ko, V. I., Colombo, L., Gellert, P. R., Schwab, M. G., and Kim, K. (2012). A roadmap for graphene. Nature 490, 192-200. doi: 10.1038/nature11458

Ou, L., Song, B., Liang, H., Liu, J., Feng, X., Deng, B., et al. (2016). Toxicity of graphene-family nanoparticles: a general review of the origins and mechanisms. Part. Fibre Toxicol. 13:57. doi: 10.1186/s12989-016-0168-y

Ozboyaci, M., Kokh, D. B., Corni, S., and Wade, R. C. (2016). Modeling and simulation of protein-surface interactions: achievements and challenges. $Q$. Rev. Biophys. 49:e4. doi: 10.1017/s0033583515000256

Pandey, P. K., Sharma, A. K., and Gupta, U. (2015). Blood brain barrier: an overview on strategies in drug delivery, realistic in vitro modeling and in vivo live tracking. Tissue Barriers 4:e1129476. doi: 10.1080/21688370.2015. 1129476

Pardridge, W. M. (2001). BBB-genomics: creating new openings for brain-drug targeting. Drug Discov. Today 6, 381-383. doi: 10.1016/s1359-6446(01)01721-4

Pardridge, W. M. (2007a). Blood-brain barrier delivery of protein and non-viral gene therapeutics with molecular Trojan horses. J. Control. Release 122, 345-348. doi: 10.1016/j.jconrel.2007.04.001

Pardridge, W. M. (2007b). Drug targeting to the brain. Pharm. Res. 24, 1733-1744. doi: 10.1007/s11095-007-9324-2

Pardridge, W. M. (2012). Drug transport across the blood-brain barrier. J. Cereb. Blood Flow Metab. 32, 1959-1972. doi: 10.1038/jcbfm.2012.126

Park, D. W., Ness, J. P., Brodnick, S. K., Esquibel, C., Novello, J., Atry, F., et al. (2018). Electrical neural stimulation and simultaneous in vivo monitoring with transparent graphene electrode arrays implanted in GCaMP6f mice. ACS Nano 12, 148-157. doi: 10.1021/acsnano.7b04321 
Patra, N., Song, Y., and Král, P. (2011). Self-assembly of graphene nanostructures on nanotubes. ACS Nano 5, 1798-1804. doi: 10.1021/nn102531h

Patra, N., Wang, B., and Král, P. (2009). Nanodroplet activated and guided folding of graphene nanostructures. Nano Lett. 9, 3766-3771. doi: 10.1021/nl9019616

Paul, A., Hasan, A., Kindi, H. A., Gaharwar, A. K., Rao, V. T., Nikkhah, M., et al. (2014). Injectable graphene oxide/hydrogel-based angiogenic gene delivery system for vasculogenesis and cardiac repair. ACS Nano 8, 8050-8062. doi: 10.1021/nn5020787

Pelin, M., Fusco, L., León, V., Martín, C., Criado, A., Sosa, S., et al. (2017). Differential cytotoxic effects of graphene and graphene oxide on skin keratinocytes. Sci. Rep. 7:40572. doi: 10.1038/srep40572

Perlmutter, J. S., and Mink, J. W. (2006). Deep brain stimulation. Annu. Rev. Neurosci. 29, 229-257. doi: 10.1146/annurev.neuro.29.051605.112824

Petri, B., Bootz, A., Khalansky, A., Hekmatara, T., Müller, R., Uhl, R., et al. (2007). Chemotherapy of brain tumour using doxorubicin bound to surfactant-coated poly(butyl cyanoacrylate) nanoparticles: revisiting the role of surfactants. J. Control. Release 117, 51-58. doi: 10.1016/j.jconrel.2006.10.015

Phillips, J. C., Braun, R., Wang, W., Gumbart, J., Tajkhorshid, E., Villa, E., et al. (2005). Scalable molecular dynamics with NAMD. J. Comput. Chem. 26, 1781-1802. doi: $10.1002 /$ jcc.20289

Picaud, S., and Sahel, J. A. (2014). Retinal prostheses: clinical results and future challenges. C. R. Biol. 337, 214-222. doi: 10.1016/j.crvi.2014.01.001

Pumera, M. (2009). Electrochemistry of graphene: new horizons for sensing and energy storage. Chem. Rec. 9, 211-223. doi: 10.1002/tcr.200900008

Pykal, M., Jurečka, P., Karlický, F., and Otyepka, M. (2016). Modelling of graphene functionalization. Phys. Chem. Chem. Phys. 18, 6351-6372. doi: $10.1039 / \mathrm{c} 5 \mathrm{cp} 03599 \mathrm{f}$

Qian, J., Wang, D., Cai, F. H., Xi, W., Peng, L., Zhu, Z. F., et al. (2012). Observation of multiphoton-induced fluorescence from graphene oxide nanoparticles and applications in in vivo functional bioimaging. Angew. Chem. Int. Ed Engl. 51, 10570-10575. doi: 10.1002/anie.201206107

Qiu, H., Sarathy, A., Leburton, J. P., and Schulten, K. (2015). Intrinsic stepwise translocation of stretched ssDNA in graphene nanopores. Nano Lett. 15, 8322-8330. doi: 10.1021/acs.nanolett.5b03963

Raghnaill, M. N., Bramini, M., Ye, D., Couraud, P. O., Romero, I. A., Weksler, B., et al. (2014). Paracrine signalling of inflammatory cytokines from an in vitro blood brain barrier model upon exposure to polymeric nanoparticles. Analyst 139, 923-930. doi: 10.1039/c3an01621h

Ragnaill, M. N., Brown, M., Ye, D., Bramini, M., Callanan, S., Lynch, I., et al. (2011). Internal benchmarking of a human blood-brain barrier cell model for screening of nanoparticle uptake and transcytosis. Eur. J. Pharm. Biopharm. 77, 360-367. doi: 10.1016/j.ejpb.2010.12.024

Rauti, R., Lozano, N., León, V., Scaini, D., Musto, M., Rago, I., et al. (2016). Graphene oxide nanosheets reshape synaptic function in cultured brain networks. ACS Nano 10, 4459-4471. doi: 10.1021/acsnano.6b00130

Re, F., Cambianica, I., Zona, C., Sesana, S., Gregori, M., Rigolio, R., et al. (2011). Functionalization of liposomes with ApoE-derived peptides at different density affects cellular uptake and drug transport across a blood-brain barrier model. Nanomedicine 7, 551-559. doi: 10.1016/j.nano.2011.05.004

Reina, G., González-Domínguez, J. M., Criado, A., Vázquez, E., Bianco, A., and Prato, M. (2017). Promises, facts and challenges for graphene in biomedical applications. Chem. Soc. Rev. 46, 4400-4416. doi: 10.1039/c7cs00363c

Ren, C., Hu, X., Li, X., and Zhou, Q. (2016). Ultra-trace graphene oxide in a water environment triggers Parkinson's disease-like symptoms and metabolic disturbance in zebrafish larvae. Biomaterials 93, 83-94. doi: 10.1016/j. biomaterials.2016.03.036

Rip, J., Schenk, G. J., and De Boer, A. G. (2009). Differential receptor-mediated drug targeting to the diseased brain. Expert Opin. Drug Deliv. 6, 227-237. doi: $10.1517 / 17425240902806383$

Robinson, J. T., Tabakman, S. M., Liang, Y., Wang, H., Casalongue, H. S., Vinh, D., et al. (2011). Ultrasmall reduced graphene oxide with high near-infrared absorbance for photothermal therapy. J. Am. Chem. Soc. 133, 6825-6831. doi: $10.1021 / \mathrm{ja} 2010175$

Rubin, L. L., Barbu, K., Bard, F., Cannon, C., Hall, D. E., Horner, H., et al. (1991). Differentiation of brain endothelial cells in cell culture. Ann. N Y Acad. Sci. 633, 420-425. doi: 10.1111/j.1749-6632.1991.tb15631.x

Sahoo, S., Bhattacharya, P., Dhibar, S., Hatui, G., Das, T., and Das, C. K. (2015). Graphene/poly(aniline-co-pyrrole) nanocomposite: potential candidate for supercapacitor and microwave absorbing applications. J. Nanosci. Nanotechnol. 15, 6931-6941. doi: 10.1166/jnn.2015.10540

Saraiva, C., Praça, C., Ferreira, R., Santos, T., Ferreira, L., and Bernardino, L. (2016). Nanoparticle-mediated brain drug delivery: overcoming blood-brain barrier to treat neurodegenerative diseases. J. Control. Release 235, 34-47. doi: 10.1016/j.jconrel.2016.05.044

Sathe, C., Girdhar, A., Leburton, J. P., and Schulten, K. (2014). Electronic detection of dsDNA transition from helical to zipper conformation using graphene nanopores. Nanotechnology 25:445105. doi: 10.1088/0957-4484/25/44/ 445105

Sathe, C., Zou, X., Leburton, J. P., and Schulten, K. (2011). Computational investigation of DNA detection using graphene nanopores. ACS Nano 5, 8842-8851. doi: $10.1021 / \mathrm{nn} 202989 \mathrm{w}$

Sayyar, S., Bjorninen, M., Haimi, S., Miettinen, S., Gilmore, K., Grijpma, D., et al. (2016). UV cross-linkable graphene/poly(trimethylene carbonate) composites for 3D printing of electrically conductive scaffolds. ACS Appl. Mater. Interfaces 8, 31916-31925. doi: 10.1021/acsami.6b09962

Seabra, A. B., Paula, A. J., de Lima, R., Alves, O. L., and Duran, N. (2014). Nanotoxicity of graphene and graphene oxide. Chem. Res. Toxicol. 27, 159-168. doi: $10.1021 / \mathrm{tx} 400385 \mathrm{x}$

Semmler-Behnke, M., Kreyling, W. G., Lipka, J., Fertsch, S., Wenk, A., Takenaka, S., et al. (2008). Biodistribution of 1.4- and 18-nm gold particles in rats. Small 4, 2108-2111. doi: 10.1002/smll.200800922

Serrano, M. C., Patiño, J., García-Rama, C., Ferrer, M. L., Fierro, J. L. G., Tamayo, A., et al. (2014). 3D free-standing porous scaffolds made of graphene oxide as substrates for neural cell growth. J. Mater. Chem. B 2, 5698-5706. doi: 10.1039/c4tb00652f

Servant, A., Bianco, A., Prato, M., and Kostarelos, K. (2014a). Graphene for multifunctional synthetic biology: the last 'zeitgeist' in nanomedicine. Bioorg. Med. Chem. Lett. 24, 1638-1649. doi: 10.1016/j.bmcl.2014.01.051

Servant, A., Leon, V., Jasim, D., Methven, L., Limousin, P., FernandezPacheco, E. V., et al. (2014b). Graphene-based electroresponsive scaffolds as polymeric implants for on-demand drug delivery. Adv. Healthc. Mater. 3, 1334-1343. doi: 10.1002/adhm.201400016

Song, Q., Jiang, Z., Li, N., Liu, P., Liu, L., Tang, M., et al. (2014). Antiinflammatory effects of three-dimensional graphene foams cultured with microglial cells. Biomaterials 35, 6930-6940. doi: 10.1016/j.biomaterials.2014. 05.002

Spelman, F. A. (2006). Cochlear electrode arrays: past, present and future. Audiol. Neurootol. 11, 77-85. doi: 10.1159/000090680

Strazielle, N., and Ghersi-Egea, J. F. (2013). Physiology of blood-brain interfaces in relation to brain disposition of small compounds and macromolecules. Mol. Pharm. 10, 1473-1491. doi: 10.1021/mp300518e

Sun, X., Feng, Z., Hou, T., and Li, Y. (2014). Mechanism of graphene oxide as an enzyme inhibitor from molecular dynamics simulations. ACS Appl. Mater. Interfaces 6, 7153-7163. doi: 10.1021/am500167c

Sun, X., Liu, Z., Welsher, K., Robinson, J. T., Goodwin, A., Zaric, S., et al. (2008). Nano-graphene oxide for cellular imaging and drug delivery. Nano Res. 1, 203-212. doi: 10.1007/s12274-008-8021-8

Tabatabaei, S. N., Girouard, H., Carret, A. S., and Martel, S. (2015). Remote control of the permeability of the blood-brain barrier by magnetic heating of nanoparticles: a proof of concept for brain drug delivery. J. Control. Release 206, 49-57. doi: 10.1016/j.jconrel.2015.02.027

Terakawa, T., Kameda, T., and Takada, S. (2011). On easy implementation of a variant of the replica exchange with solute tempering in GROMACS. J. Comput. Chem. 32, 1228-1234. doi: 10.1002/jcc.21703

Thompson, B. C., Murray, E., and Wallace, G. G. (2015). Graphite oxide to graphene. Biomaterials to bionics. Adv. Mater. 27, 7563-7582. doi: 10.1002/adma.201500411

Tian, X., Yang, Z., Duan, G., Wu, A., Gu, Z., Zhang, L., et al. (2017). Graphene oxide nanosheets retard cellular migration via disruption of actin cytoskeleton. Small 13:1602133. doi: 10.1002/smll.201602133

Titov, A. V., Král, P., and Pearson, R. (2010). Sandwiched graphene-membrane superstructures. ACS Nano 4, 229-234. doi: 10.1021/nn9015778

Tonelli, F. M., Goulart, V. A., Gomes, K. N., Ladeira, M. S., Santos, A. K., Lorencon, E., et al. (2015). Graphene-based nanomaterials: biological and medical applications and toxicity. Nanomedicine 10, 2423-2450. doi: $10.2217 / \mathrm{nnm} .15 .65$ 
Tosi, G., Costantino, L., Ruozi, B., Forni, F., and Vandelli, M. A. (2008). Polymeric nanoparticles for the drug delivery to the central nervous system. Expert Opin. Drug Deliv. 5, 155-174. doi: 10.1517/17425247.5.2.155

Treat, L. H., McDannold, N., Vykhodtseva, N., Zhang, Y., Tam, K., and Hynynen, K. (2007). Targeted delivery of doxorubicin to the rat brain at therapeutic levels using MRI-guided focused ultrasound. Int. J. Cancer 121, 901-907. doi: 10.1002/ijc.22732

Tu, Y., Lv, M., Xiu, P., Huynh, T., Zhang, M., Castelli, M., et al. (2013). Destructive extraction of phospholipids from Escherichia coli membranes by graphene nanosheets. Nat. Nanotechnol. 8, 594-601. doi: 10.1038/nnano. 2013.125

Tu, Q., Pang, L., Chen, Y., Zhang, Y., Zhang, R., Lu, B., et al. (2014). Effects of surface charges of graphene oxide on neuronal outgrowth and branching. Analyst 139, 105-115. doi: 10.1039/c3an01796f

Ulbrich, K., Hekmatara, T., Herbert, E., and Kreuter, J. (2009). Transferrinand transferrin-receptor-antibody-modified nanoparticles enable drug delivery across the blood-brain barrier (BBB). Eur. J. Pharm. Biopharm. 71, 251-256. doi: 10.1016/j.ejpb.2008.08.021

Ulbrich, K., Knobloch, T., and Kreuter, J. (2011). Targeting the insulin receptor: nanoparticles for drug delivery across the blood-brain barrier (BBB). J. Drug Target. 19, 125-132. doi: 10.3109/10611861003734001

Ulloa Severino, F. P., Ban, J., Song, Q., Tang, M., Bianconi, G., Cheng, G., et al. (2016). The role of dimensionality in neuronal network dynamics. Sci. Rep. 6:29640. doi: 10.1038/srep29640

Upadhyay, R. K. (2014). Drug delivery systems, CNS protection and the blood brain barrier. Biomed Res. Int. 2014:869269. doi: 10.1155/2014/ 869269

van Rooy, I., Mastrobattista, E., Storm, G., Hennink, W. E., and Schiffelers, R. M. (2011). Comparison of five different targeting ligands to enhance accumulation of liposomes into the brain. J. Control. Release 150, 30-36. doi: 10.1016/j. jconrel.2010.11.014

Veliev, F., Han, Z., Kalita, D., Briançon-Marjollet, A., Bouchiat, V., and Delacour, C. (2017). Recording spikes activity in cultured hippocampal neurons using flexible or transparent graphene transistors. Front. Neurosci. 11:466. doi: 10.3389/fnins.2017.00466

Wagner, S., Zensi, A., Wien, S. L., Tschickardt, S. E., Maier, W., Vogel, T., et al. (2012). Uptake mechanism of ApoE-modified nanoparticles on brain capillary endothelial cells as a blood-brain barrier model. PLoS One 7:e32568. doi: 10.1371/journal.pone.0032568

Wang, Y., Li, Z., Hu, D., Lin, C. T., Li, J., and Lin, Y. (2010). Aptamer/graphene oxide nanocomplex for in situ molecular probing in living cells. J. Am. Chem. Soc. 132, 9274-9276. doi: 10.1021/ja103169v

Wang, X., Sun, X., Lao, J., He, H., Cheng, T., Wang, M., et al. (2014). Multifunctional graphene quantum dots for simultaneous targeted cellular imaging and drug delivery. Colloids Surf. B Biointerfaces 122, 638-644. doi: 10.1016/j.colsurfb.2014.07.043

Wang, D. C., Zhang, Y., Chen, H. Y., Li, X. L., Qin, L. J., Li, Y. J., et al. (2012). Hyperthermia promotes apoptosis and suppresses invasion in C6 rat glioma cells. Asian Pac. J. Cancer Prev. 13, 3239-3245. doi: 10.7314/apjcp.2012.13. 7.3239

Wells, D. B., Belkin, M., Comer, J., and Aksimentiev, A. (2012). Assessing graphene nanopores for sequencing DNA. Nano Lett. 12, 4117-4123. doi: $10.1021 / \mathrm{nl} 301655 \mathrm{~d}$

Wick, P., Louw-Gaume, A. E., Kucki, M., Krug, H. F., Kostarelos, K., Fadeel, B., et al. (2014). Classification framework for graphene-based materials. Angew. Chem. Int. Ed Engl. 53, 7714-7718. doi: 10.1002/anie.2014 03335

Willems, N., Urtizberea, A., Verre, A. F., Iliut, M., Lelimousin, M., Hirtz, M., et al. (2017). Biomimetic phospholipid membrane organization on graphene and graphene oxide surfaces: a molecular dynamics simulation study. ACS Nano 11, 1613-1625. doi: 10.1021/acsnano.6b07352

Wolburg, H., and Lippoldt, A. (2002). Tight junctions of the blood-brain barrier: development, composition and regulation. Vascul Pharmacol. 38, 323-337. doi: 10.1016/S1537-1891(02)00200-8

Xiao, S., Zhou, D., Luan, P., Gu, B., Feng, L., Fan, S., et al. (2016). Graphene quantum dots conjugated neuroprotective peptide improve learning and memory capability. Biomaterials 106, 98-110. doi: 10.1016/j.biomaterials.2016. 08.021
Xu, X., Wang, J., Wang, Y., Zhao, L., Li, Y., and Liu, C. (2017). Formation of graphene oxide-hybridized nanogels for combinative anticancer therapy. Nanomedicine doi: 10.1016/j.nano.2017.05.007 [Epub ahead of print].

Yang, K., Hu, L., Ma, X., Ye, S., Cheng, L., Shi, X., et al. (2012). Multimodal imaging guided photothermal therapy using functionalized graphene nanosheets anchored with magnetic nanoparticles. Adv. Mater. 24, 1868-1872. doi: 10.1002/adma.201104964

Yang, K., Feng, L., Shi, X., and Liu, Z. (2013). Nano-graphene in biomedicine: theranostic application. Chem. Soc. Rev. 42, 530-547. doi: 10.1039/c2cs35342c

Yang, H. W., Huang, C. Y., Lin, C. W., Liu, H. L., Huang, C. W., Liao, S. S., et al. (2014). Gadolinium-functionalized nanographene oxide for combined drug and microRNA delivery and magnetic resonance imaging. Biomaterials 35, 6534-6542. doi: 10.1016/j.biomaterials.2014.04.057

Yang, L., Wang, F., Han, H., Yang, L., Zhang, G., and Fan, Z. (2015). Functionalized graphene oxide as a drug carrier for loading pirfenidone in treatment of subarachnoid hemorrhage. Colloids Surf. B Biointerfaces 129, 21-29. doi: 10.1016/j.colsurfb.2015.03.022

Yang, W., Zhao, Y., He, X., Chen, Y., Xu, J., Li, S., et al. (2015). Flexible conducting polymer/reduced graphene oxide films: synthesis, characterization and electrochemical performance. Nanoscale Res. Lett. 10:222. doi: 10.1186/s11671015-0932-1

Yang, K., Zhang, S., Zhang, G., Sun, X., Lee, S. T., and Liu, Z. (2010). Graphene in mice: ultrahigh in vivo tumor uptake and efficient photothermal therapy. Nano Lett. 10, 3318-3323. doi: 10.1021/nl100996u

Yeo, J., Cheng, Y., Han, Y. T., Zhang, Y., Guan, G., and Zhang, Y. W. (2016). Adsorption and conformational evolution of $\alpha$-helical BSA segments on graphene: a molecular dynamics study. Int. J. Appl. Mech. 8:1650021. doi: $10.1142 / \mathrm{s} 1758825116500216$

Zeng, S., Zhou, G., Guo, J., Zhou, F., and Chen, J. (2016). Molecular simulations of conformation change and aggregation of HIV-1 Vpr13-33 on graphene oxide. Sci. Rep. 6:24906. doi: 10.1038/srep24906

Zensi, A., Begley, D., Pontikis, C., Legros, C., Mihoreanu, L., Büchel, C., et al. (2010). Human serum albumin nanoparticles modified with apolipoprotein A-I cross the blood-brain barrier and enter the rodent brain. J. Drug Target. 18, 842-848. doi: 10.3109/1061186X.2010.513712

Zensi, A., Begley, D., Pontikis, C., Legros, C., Mihoreanu, L., Wagner, S., et al. (2009). Albumin nanoparticles targeted with Apo E enter the CNS by transcytosis and are delivered to neurones. J. Control. Release 137, 78-86. doi: 10.1016/j.jconrel.2009.03.002

Zhang, Y., Ali, S. F., Dervishi, E., Xu, Y., Li, Z., Casciano, D., et al. (2010). Cytotoxicity effects of graphene and single-wall carbon nanotubes in neural phaeochromocytoma-derived PC12 cells. ACS Nano 4, 3181-3186. doi: $10.1021 / \mathrm{nn} 1007176$

Zhang, H., Gruner, G., and Zhao, Y. (2013). Recent advancements of graphene in bomedicine. J. Mater. Chem. B 1, 2542-2567. doi: 10.1039/c3tb20405g

Zhang, L., Lu, Z., Zhao, Q., Huang, J., Shen, H., and Zhang, Z. (2011). Enhanced chemotherapy efficacy by sequential delivery of siRNA and anticancer drugs using PEI-grafted graphene oxide. Small 7, 460-464. doi: 10.1002/smll. 201001522

Zhang, X., Yin, J., Peng, C., Hu, W., Zhu, Z., Li, W., et al. (2011). Distribution and biocompatibility studies of graphene oxide in mice after intravenous administration. Carbon 49, 986-995. doi: 10.1016/j.carbon.2010.11.005

Zhang, Q., Wu, Z., Li, N., Pu, Y., Wang, B., Zhang, T., et al. (2017). Advanced review of graphene-based nanomaterials in drug delivery systems: synthesis, modification, toxicity and application. Mater. Sci. Eng. C Mater. Biol. Appl. 77, 1363-1375. doi: 10.1016/j.msec.2017.03.196

Zhang, D., Zhang, Z., Liu, Y., Chu, M., Yang, C., Li, W., et al. (2015). The short- and long-term effects of orally administered high-dose reduced graphene oxide nanosheets on mouse behaviors. Biomaterials 68, 100-113. doi: 10.1016/j. biomaterials.2015.07.060

Zhao, Y., Gong, J., Niu, C., Wei, Z., Shi, J., Li, G., et al. (2017). A new electrospun graphene-silk fibroin composite scaffolds for guiding Schwann cells. J. Biomater. Sci. Polym. 28, 2171-2185. doi: 10.1080/09205063.2017. 1386835

Zhao, Y., Li, Z., Sharma, U. K., Sharma, N., Song, G., and Van der Eycken, E. V. (2016). Copper-catalyzed alkylarylation of activated alkenes using isocyanides as the alkyl source: an efficient radical access to 3,3-dialkylated oxindoles. Chem. Commun. 52, 6395-6398. doi: 10.1039/c6cc02024k 
Zhao, X., Shang, T., Zhang, X., Ye, T., Wang, D., and Rei, L. (2016). Passage of Magnetic tat-conjugated $\mathrm{Fe}_{3} \mathrm{O}_{4} @ \mathrm{SiO}_{2}$ nanoparticles across in vitro blood-brain barrier. Nanoscale Res. Lett. 11:451. doi: 10.1186/s11671-0161676-2

Zhou, K., Motamed, S., Thouas, G. A., Bernard, C. C., Li, D., Parkington, H. C., et al. (2016). Graphene functionalized scaffolds reduce the inflammatory response and supports endogenous neuroblast migration when implanted in the adult brain. PLoS One 11:e0151589. doi: 10.1371/journal.pone. 0151589

Zhou, H., Zhao, K., Li, W., Yang, N., Liu, Y., Chen, C., et al. (2012). The interactions between pristine graphene and macrophages and the production of cytokines/chemokines via TLR- and NF- $\mathrm{B}$-related signaling pathways. Biomaterials 33, 6933-6942. doi: 10.1016/j.biomaterials.2012. 06.064

Zhu, C., Han, T. Y., Duoss, E. B., Golobic, A. M., Kuntz, J. D., Spadaccini, C. M., et al. (2015). Highly compressible 3D periodic graphene aerogel microlattices. Nat. Commun. 6:6962. doi: 10.1038/ncomms7962

Zuccaro, L., Tesauro, C., Kurkina, T., Fiorani, P., Yu, H. K., Knudsen, B. R., et al. (2015). Real-time label-free direct electronic monitoring of topoisomerase enzyme binding kinetics on graphene. ACS Nano 9, 11166-11176. doi: 10.1021/acsnano.5b05709

Zuo, G., Zhou, X., Huang, Q., Fang, H., and Zhou, R. (2011). Adsorption of Villin headpiece onto graphene, carbon nanotube and C60: effect of conctacting surface curvatures on binding affinity. J. Phys. Chem. C 115, 23323-23328. doi: $10.1021 /$ jp208967t

Conflict of Interest Statement: The authors declare that the research was conducted in the absence of any commercial or financial relationships that could be construed as a potential conflict of interest.

Copyright (C) 2018 Bramini, Alberini, Colombo, Chiacchiaretta, DiFrancesco, MayaVetencourt, Maragliano, Benfenati and Cesca. This is an open-access article distributed under the terms of the Creative Commons Attribution License (CC BY). The use, distribution or reproduction in other forums is permitted, provided the original author(s) and the copyright owner are credited and that the original publication in this journal is cited, in accordance with accepted academic practice. No use, distribution or reproduction is permitted which does not comply with these terms. 